\title{
EL SOLAR MAYA-CH'OL Y SUS SABERES ETNOBOTÁNICOS EN COMUNIDADES AL NORTE DE CHIAPAS, MÉXICO
}

\author{
THE MAYAN-CH'OL SITE AND ETHNOBOTANICAL KNOWLEDGE IN COMMUNITIES \\ NORTH OF CHIAPAS, MEXICO
}

\author{
${ }^{1 *}$ Paola Andrea UBIERGO-CORVALÁN, ${ }^{2}$ Guadalupe RODRÍGUEZ-GALVÁN, ${ }^{3}$ Mercedes CASTRO-LAPORTTE, ${ }^{4}$ Lourdes \\ ZARAGOZA-MARTÍNEZ, ${ }^{5}$ Alejandro CASAS, ${ }^{6}$ Francisco GUEVARA-HERNÁNDEZ
}

\begin{abstract}
${ }^{1}$ Universidad Autónoma de Chiapas (UNACHEstudiante de Doctorado en Ciencias y Sustentabilidad (DOCAS); ${ }^{2}$ Universidad Autónoma de Chiapas; Instituto de Estudios Indígenas; ${ }^{3}$ Universidad Central de Venezuela;Facultad de Agronomía; Instituto de Botánica Agrícola; ${ }^{4}$ Universidad Autónoma de Chiapas; Escuela en Ciencias y Procesos Agroindustriales; ${ }^{5}$ Universidad Nacional Autónoma de México; Instituto de Investigaciones en Ecosistemas y Sustentabilidad;

${ }^{6}$ Universidad Autónoma de Chiapas;Facultad de Ciencias Agronómicas; *paola.ubiergo@gmail.com
\end{abstract}

\section{RESUMEN}

El agroecosistema denominado solar presenta un papel fundamental en el sistema de vida del pueblo ch'ol en el sureste de México; la conservación del conocimiento tradicional de especies vegetales, ha permitido obtener diversos recursos para la subsistencia y han contribuido en los ingresos económicos de las familias. Por ello, los objetivos de esta investigación fueron describir el agroecosistema denominado "solar", identificar las especies vegetales que las familias choles utilizan y determinar las categorías antropocéntricas del uso de estas especies, con base a sus conocimientos tradicionales. Esta investigación se realizó en comunidades maya-ch'ol del Valle del Tulijá, al norte de Chiapas, México; para la obtención de la información, se implementó el proceso metodológico SIV (Sistemas de Vida), se aplicaron herramientas participativas y técnicas tradicionales de muestreo etnobotánico, en el periodo agosto 2016 a agosto 2017; el análisis de la información fue desarrollado mediante métodos descriptivos. El solar maya-ch'ol se caracteriza por presentar cuatro espacios zonificados destinados para el aprovechamiento de sus recursos, los cuales son traspatio, hortaliza, jardín y reserva forestal; en los cuales se registran 131 especies útiles, agrupadas en 58 familias, sobre todo representadas por Fabaceae (11 especies), Malvaceae y Rutaceae (8 especies); de todas las especies destaca el registro de 65 nombres de plantas en lengua ch'ol. Del total de especies, se presenta una clasificación de 19 categorías de usos antropocéntricos, representados en su mayoría por uso comestible, medicinal y ornamental; asimismo, los biotipos usados con mayor frecuencia son los árboles, y las partes más utilizadas son los frutos y las hojas. Bajo tal contexto, este estudio permitió revalorizar los saberes etnobotánicos en relación al manejo de los componentes del solar; además, se hace evidente la importancia de este espacio en el ámbito del sistema de vida del pueblo maya-ch'ol.

PALABRAS CLAVE: agrobiodiversidad; agroecosistemas; conocimientos tradicionales; huertos familiares; pueblos indígenas

\begin{abstract}
The agroecosystem called solar presents a fundamental role in the way of life of the ch'ol people in the southeast of Mexico; the preservation of traditional knowledge of plant species has allowed obtaining diverse resources for livelihood and has contributed to the economic income of families. Therefore, the objectives of this research were to describe the agroecosystem called "solar", identify the plant species that the ch'ol families use and determine the anthropocentric categories of the use of these species, based on their traditional knowledge. This research was conducted in Mayan ch'ol communities of the Tulijá Valley, north of Chiapas, Mexico; in order to obtain the information, the methodological process SIV (Life Systems) was implemented, participatory tools and traditional ethnobotanical sampling techniques were applied, from August 2016 to August 2017; the analysis of the information was developed by descriptive methods. The Mayan solar ch'ol is characterized by four zoned spaces intended for the use of its resources, which are backyard, vegetable zone, garden and forest reserve; in which 131 useful species are recorded, grouped in 58 families, represented by Fabaceae (11 species), Malvaceae and Rutaceae ( 8 species); of all the species, the record of 65 plant names in the $c h$ 'ol language stand out. Of the total of species, a classification of 19 categories of anthropocentric uses is presented, represented in their majority by edible, medicinal and ornamental use; also, the most commonly used biotypes are trees, and the most commonly used parts are fruits and leaves. In this context, this study allowed us to revalue ethnobotanical knowledge in relation to the management of solar components; in addition, the importance of this field in the Mayan-ch'ol people's way of life becomes evident.
\end{abstract}

KEYWORDS: agrobiodiversity, agroecosystems, traditional knowledge, family gardens, indigenous people 


\section{INTRODUCCIÓN \\ El agroecosistema solar}

Los sistemas agrícolas tradicionales constituyen un aporte económico vital para la sobrevivencia de las comunidades rurales. En éstos, se desarrollan espacios de conservación de recursos naturales bajo prácticas agrícolas, para asegurar requerimientos equilibrados en sus sistemas de vida (ALTIERI y NICHOLLS, 2000; MENDOZA, 2015; MONTENEGRO et al., 2017).

Se ha demostrado que en los agroecosistemas familiares existen vínculos entre la diversidad vegetal, el aporte nutricional y la salud; debido a que las especies culturalmente significativas tienen importancia social y biológica en la salud de la población. Además, constituyen la principal fuente de material genético para elaborar productos biotecnológicos (JOHNS, 2011; HERNÁNDEZ-RUÍZ et al., 2014; QUINTERO et al., 2015).

En Latinoamérica existe un alto grado de diversidad vegetal representado como policultivos; lo cual es uno de los importantes desafíos en la compresión del modo en que los campesinos preservan y manejan la biodiversidad. Basado en esto, se reconoce la complejidad de sus unidades productivas, relacionadas con los conocimientos tradicionales de quienes las manejan (ALTIERI y NICHOLLS, 2000; QUINTERO et al., 2015; MENDOZA, 2015; MONTENEGRO et al., 2017).

México presenta una importante riqueza en conocimientos prehispánicos sobre especies vegetales seleccionadas, domesticadas y conservadas dentro de agroecosistemas tradicionales (HERNÁNDEZ-XOLOCOTZI, 1998; LUNA-MORALES, 2002; VENEGAS, 2017); asociado al hecho de que es considerado el tercer país megadiverso y centro de origen de diversas especies de importancia agrícola (CONABIO, 2015). Algunos estudios señalan la riqueza del conocimiento agrícola prehispánico y arqueobotánico del uso de las plantas (MELÉNDEZ et al., 2012; VENEGAS, 2017); no obstante, se ha demostrado que este conocimiento aún constituye un recurso potencial poco conocido, a nivel cognoscitivo y tecnológico (LUNA-MORALES, 2002).

Basado en esto, se considera que entender el desarrollo social implica "estudiar los conocimientos indígenas botánicos y agrícolas" (HERNÁNDEZ-XOLOCOTZI, 1998). Todo lo anterior, amerita apoyo para generar trabajos de investigación y promover programas apropiados (RODRÍGUEZ-GALVÁN, 2011; FARRERA y ORANTES, 2015).

Chiapas es uno de los estados de México con mayor diversidad florística y étnica, habitan nueve grupos indígenas y presenta una riqueza aproximadamente de 10000 especies vegetales (CONABIO, 2015; FARRERA Y ORANTES, 2015). Este patrimonio ha permitido que exista un importante auge de conocimientos sobre formas de uso y manejo de los recursos vegetales (RUBIODELGADO, 2013; HERNÁNDEZ, 2013; ALEMÁN-SANTILLÁN, 2013). Particularmente los bosques tropicales de Chiapas, han provisto a las comunidades rurales de una complejidad de recursos naturales que dan sentido a su identidad étnica. Además, las familias aprovechan esta diversidad para la subsistencia y conservación de prácticas tradicionales, mediante unidades de producción, que van de acuerdo con la situación social, económica y estrategias productivas (HERNÁNDEZ, 2013; ALEMÁN-SANTILLÁN, 2013).

El agroecosistema denominado "solar" presenta un papel fundamental en el sistema de vida de estas poblaciones (MARIACA, 2012). El manejo tradicional ha permitido generar el uso y aprovechamiento de especies para la subsistencia familiar, para la obtención de diversos recursos vegetales; también, se le atribuye el impacto productivo a pequeña escala para generar ingresos económicos; por tanto ha sido importante en los aspectos de la vida social y cultural (RODRÍGUEZGALVÁN, 2011; MARIACA, 2012; CABALLERO-ROQUE et al., 2018).

\section{El pueblo indígena maya-ch'ol}

Al norte de Chiapas, uno de los grupos indígenas mejor representados en una gran extensión de este territorio es el pueblo maya-ch'ol. Su población se encuentra asentada en un área originalmente cubierta por bosques tropicales perennifolios y bosques mesófilos de montaña; área propicia para el desarrollo de estudios etnobiológicos, ya que actualmente presenta de gran vulnerabilidad debido al impacto de las actividades antropogénicas (MORALES y SALVATIERRA, 2012; AGUILAR, 2014; PIGNATARO et al., 2016).

Por su parte, la economía de este pueblo está basada en la agricultura de subsistencia, presentan un sistema agrícola integral, basado en la producción de maíz y su interacción con agroecosistemas forestales; por lo cual, el maíz y el frijol son los cultivos de mayor importancia para autoabasto, y el café para la obtención de ingresos (LERNER et al., 2009; AGUILAR, 2014). 
Asimismo, presentan sistemas agropecuarios comerciales, como la ganadería y el cultivo de Palma Aceitera (MORALES y SALVATIERRA, 2012; AGUILAR, 2014; PIGNATARO et al., 2016).

Sobre el pueblo ch'ol de Chiapas se han realizado diversos estudios agroecológicos; sin embargo, son pocos los que se han referido a la etnobotánica de los huertos familiares (LERNER et al., 2009; AGUIRRE-RUÍZ et al., 2013; CABALLERO-ROQUE et al., 2018); aunque, principalmente han sido orientados al manejo del sistema de la milpa ch'ol (AGUILAR, 2014).

Por otra parte, en una actualización de datos etnobiológicos sobre lenguas indígenas de México, De Ávila (2008) señala que "no se presentaba información etnobiológica sobre la etnia ch'ol, salvo datos que aparecen en diccionarios de lengua maya ch'ol de Tila (SCHUMANN, 1973), Tumbalá (AULIE y AULIE, 2009), la recopilación de Montejo et al. (2001) y el material de Kaufman y Justeson (2003)".

Sin embargo, existen investigaciones etnobotánicas como la de Vogl et al. (2002), quienes realizaron un estudio en huertos familiares ch'ol-tzeltal y mestizo en comunidades de Palenque, donde señalan 241 especies útiles. Posteriormente, Lerner et al. (2009), estudian el huerto familiar ch'ol como aporte de alimentos a su economía, realizan una clasificación socioeconómica e históricacultural y señalan al huerto como único espacio de producción y fuente principal de plantas alimenticias para familias de estratos bajos. Seguidamente, Aguirre-Ruíz et al. (2013) caracterizan los traspatios en una comunidad de Yajalón, donde contemplan mayor frecuencia de uso de 19 especies de plantas medicinales y señalan la importancia de la medicina tradicional para la comunidad.

Lo anterior hace evidente, la necesidad de proponer el rescate de los conocimientos tradicionales que la etnia ch'ol de Chiapas ha acumulado durante años sobre las especies vegetales, y resaltar la importancia de los saberes etnobotánicos para sostener sus sistemas productivos. Basado en esto, se planteó el objetivo de reconocer los diversos ambientes presentes en el solar, e identificar las especies vegetales útiles presentes en este agroecosistema, en comunidades maya-ch'ol del Valle del Tulijá, Chiapas; de manera de, contribuir a la recuperación y conservación de los saberes etnobotánicos del pueblo ch'ol y su relación con los agroecosistemas del Valle del Tulijá, Chiapas.

\section{MATERIALES Y MÉTODOS Área de estudio}

Este estudio se realizó en tres comunidades (Arroyo Encanto, Generación 95 y Rubí Tulijá), localizadas en el Valle del Tulijá al noroeste del estado de Chiapas, México; entre las coordenadas $17^{\circ} 10^{\prime} \mathrm{N}-17^{\circ} 30^{\prime} \mathrm{N}$ y $92^{\circ} 00^{\prime} \mathrm{O}-92^{\circ} 25^{\prime} \mathrm{O}$, desde 60 a $800 \mathrm{~m}$ de altitud y con una extensión territorial de $1289,20 \mathrm{~km}^{2}$ (Figura 1).

La zona de estudio pertenece al municipio Salto de Agua, de la Región Económica XIV Tulijá Tseltal Chol, limita al norte con el estado de Tabasco y el municipio Palenque, al sur colinda con los municipios Chilón y Tumbalá, y al oeste con Tila; entre las provincias fisiográficas de la Planicie Costera Suroriental y el Macizo Central de Chiapas, ubicada en la Subprovincia Fisiográfica Sierra Lacandona (RZEDOWSKI, 2006; CDI, 2012; INEGI, 2017).

En esta región, la temporada de lluvias se prolonga nueve meses, los escurrimientos forman numerosos arroyos y ríos, entre los cuales destacan Tulijá, Ixtialjá, Chinal y Pulpitillo, todos los cuales desembocan en el río Usumacinta (ANDRADE y MEJÍA, 2015)

El territorio del Valle del Tulijá presenta una población total de 5,510 habitantes, de la cual $99.44 \%$ es población indígena de origen mayense, de las etnias ch'ol (95\%) y tzeltal (5\%). Aunque la mayoría de la población es migrante de los municipios Tila y Tumbalá, aún en su mayoría preservan sus actividades tradicionales para su subsistencia (MORALES y SALVATIERRA, 2012; CDI, 2012).

En este territorio la economía se fundamenta en las actividades agropecuarias y los procesos productivos primarios se basan en la economía campesina, en donde la mano de obra familiar constituye la principal fuerza de trabajo. La ganadería extensiva para pie de cría y bovinos de engorda, la siembra de maíz y frijol y la palma aceitera son las actividades principales, y en menor proporción la ganadería de traspatio, así como los cultivos de frutales (cítricos) y algunas hortalizas (CDI, 2012; MORALES y SALVATIERRA, 2012; AGUILAR, 2014; PIGNATARO et al., 2016). 


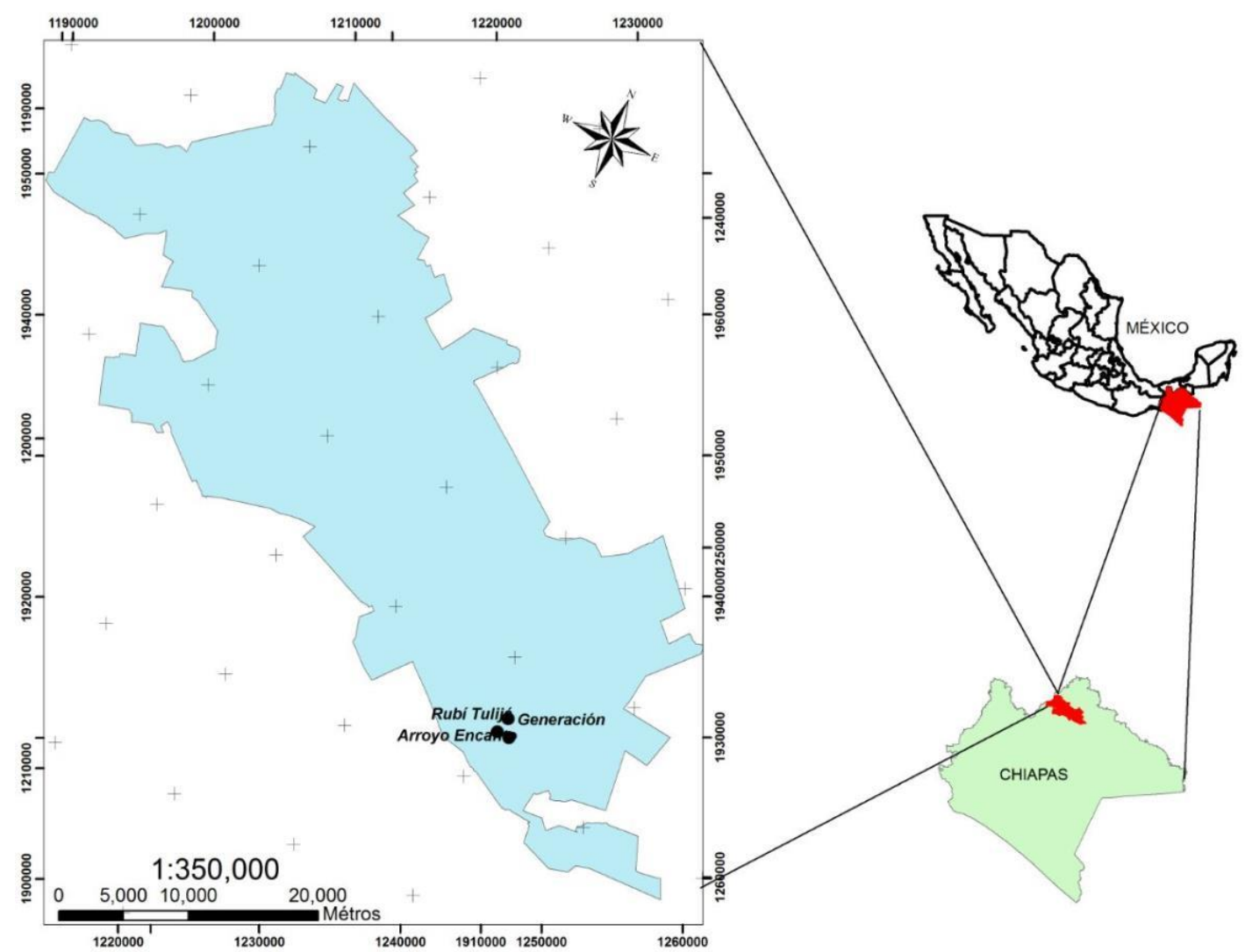

Figura 1: Ubicación geográfica de las localidades de estudio, Valle del Tulijá, Chiapas, México (Fuente INEGI, 2017).

\section{Obtención de la información}

La recopilación de datos se estableció en el periodo de agosto 2016 a agosto 2017, mediante el proceso metodológico SIV (Sistemas de Vida) estandarizado en el Instituto de Estudios Indígenas de la Universidad Autónoma de Chiapas; el cual se basa en el enfoque cualitativo, e integra un grupo de herramientas metodológicas participativas que dependen del contexto de la investigación (RODRÍGUEZ-GALVÁN et al., 2015; MENDOZA, 2015).

Se realizó el contacto inicial con la autoridad local (Comisariado ejidal) para plantear los objetivos del trabajo, con la colaboración de estudiantes de licenciatura de la Facultad Maya de Estudios Agropecuarios de la UNACH quienes apoyaron como traductores. En reunión con los comisariados se analizó la situación de la comunidad, se aprobó y acordó el trabajo de encuestas con el consentimiento de las familias.

Se aplicó una cédula informativa comunitaria a las autoridades locales de cada comunidad, para recabar datos del contexto socioterritorial. Para conocer las características del solar, a nivel cultural, socioeconómico y biológico, se implementó una encuesta al $30 \%$ de las familias, mediante el método de muestreo no probabilístico "bola de nieve" (SANDOVAL, 2002).

Se identificaron informantes clave, a quienes se les realizó una entrevista abierta a profundidad, y fueron acompañantes de las caminatas etnobotánicas en los solares, para identificar y colectar especímenes representativos de las plantas útiles. Además, se utilizó la técnica de observación participante para recoger datos sobre el manejo y otros elementos que intervienen en el sistema del solar (VOGL et al., 2004).

\section{Análisis de la información}

Con los datos de las encuestas sobre los solares, se realizó una descripción cualitativa del sistema general, los componentes y la zonificación que se presenta (AGUIRRE y JARAMILLO, 2015). Se consideraron las categorías antropocéntricas de uso de las plantas registradas por los grupos informantes, y fueron definidas como aquellas donde el grupo humano da un valor utilitario a las especies (TOLEDO et al., 1995; LEVY et al., 2002; HERNÁNDEZ-RUÍZ et al., 2014). 
Las especies enlistadas se determinaron taxonómicamente, y se emplearon diversas referencias de identificación (consulta de material de herbario, floras, bases de datos florísticos, guías de plantas útiles y consulta a especialistas). Las muestras fueron depositadas en las colecciones existentes de especímenes de herbario de la UNACH (Facultad de Ciencias Agronómicas) y el Herbario de la Universidad Juárez Autónoma de Tabasco (UJAT).

Para el análisis cuantitativo de la información suministrada por los habitantes de la comunidad, se aplicaron sumatoria de usos totales, sumatoria de frecuencias y valor de uso de las especies registradas (MARÍN-CORBA et al., 2005). Se empleó el enfoque de consenso de informantes, metodología desarrollada por Phillips (1996); valor que expresa la importancia o valor cultural de una especie determinada para todos los informantes entrevistados. Para estimar el índice de valor de uso general de cada especie para todos los informantes (IVUs), se utilizó la fórmula $(I V U s=$ $\left.\sum_{i} U V i s / n_{s}\right)$; donde: $U V i s=$ número de usos mencionados por cada informante $(i)$, para cada especie (s), $n s=$ número de informantes entrevistados.

\section{RESULTADOS \\ Descripción del solar maya-ch'ol del Valle del Tulijá, Chiapas}

El solar maya-ch'ol es un agroecosistema donde intervienen diversos componentes, que contribuyen en la integración de actividades de producción y aprovechamiento de los recursos, para generar autoabastecimiento y conservación de germoplasma; constituido por un espacio de convivencia, organización y rol de trabajo familiar. En las comunidades del Valle del Tulijá, los solares son terrenos parcelados para uso familiar y forman parte de la comunidad en su conjunto, su distribución es compacta y continúa a los lados de la calle, y generalmente su acceso es por vías de terracería.

El terreno de los solares en general es plano o menor a $10 \%$ de pendiente, ubicados a una altitud entre $70-90 \mathrm{~m}$. El área del solar se encuentra definido entre $500-1000 \mathrm{~m}^{2}(53.9 \%)$ y $1600-1700$ $\mathrm{m}^{2}(23.1 \%)$; algunos pocos solares más aislados presentan áreas mayores a $5000 \mathrm{~m}^{2}(7.7 \%)$ y algunos dentro de la comunidad están parcelados en áreas menores con $500 \mathrm{~m}^{2}(15.4 \%)$. El terreno presenta diversos ambientes, suele estar delimitado con cercos vivos de plantas ornamentales, sembradas por estacas y mantenidas con poda de formación, con la finalidad de resguardar animales, colgar la ropa para secar y cubrir el área de convivencia familiar.

La vivienda familiar o casa habitación de la familia ch'ol se encuentra constituida por la cocina, baño y espacios para chiquero y bodega. Es un espacio de convivencia donde se realizan las tareas o actividades diarias, su infraestructura cumple la función de refugio para los miembros de la familia, funciona como dormitorio, recibidor, lugar de trabajo y reuniones. La cocina generalmente está ubicada en la zona trasera o a un costado de la casa, su construcción es a base de tablas de madera, techo de lámina de metal y piso de tierra; es el área de trabajo de las mujeres, generalmente se observa la entrada libre de las gallinas, y se ubican para el resguardo de su incubación.

La familia ch'ol presenta una estructura que se compone principalmente de un núcleo conyugal y de familias colectivas. El rol de trabajo en el solar, se caracteriza por las actividades específicas para cada miembro de la familia, así la mujer madre y jefa de familia se reconoce con el rol principal (54\%), se encarga del mantenimiento general, conservación de los componentes y aprovechamiento de los recursos del solar, es responsable de los animales, del manejo uso y cuidado de las plantas cultivadas, así como también, de elaborar los alimentos, y auxiliar en la salud de la familia.

El hombre padre y jefe de familia presenta un rol importante (29\%), apoya principalmente en las actividades de construcción, de la vivienda y del subsistema agropecuario. Por otro lado, el rol de trabajo de los hijos (17\%) generalmente está definido con base en su condición de género; son los que apoyan y acompañan en diferentes actividades rutinarias junto a los padres, como el manejo de animales, elaboración de alimentos, construcción, mantenimiento general, actividades que apoyan a su formación y conocimiento de trabajo familiar.

\section{Zonificación del solar}

Según las encuestas y la observación en campo, en el solar se presentan cuatro espacios bien zonificados, señalados como el traspatio; el huerto, hortaliza o "corral" de plantas; el jardín y la reserva forestal, destinados al aprovechamiento, manejo y conservación de los recursos.

El traspatio, está ubicado detrás de la vivienda, centrado entre la cocina y los límites que colindan con los vecinos; presenta un área aproximadamente de $20 \mathrm{~m}^{2}$, y pocas veces se observa delimitado. Se 
presentan árboles comestibles, generalmente frutales, que a su vez ofrecen sombra y se utilizan para la leña; también esta zona se utiliza para la crianza de animales domésticos, principalmente aves (gallinas, guajolotes y patos) y cerdos.

La hortaliza o huerto, también se menciona como "corral de plantas", está ubicado a un costado de la cocina; presenta un área aproximadamente de $15-20 \mathrm{~m}^{2}$, delimitada con cercas vivas para evitar la entrada de los animales domésticos. Se presentan hierbas y arbustos comestibles, medicinales y ornamentales, generalmente cultivados.

El jardín, está ubicado frente a la vivienda, a un costado de la puerta principal; presenta un área aproximadamente de $10 \mathrm{~m}^{2}$, con cercado continuo. Se presentan hierbas en maceta y arbustos cultivados como ornamentales o medicinales.

La reserva forestal, está ubicada al final del solar, colinda con solares aledaños; presenta un área aproximadamente de $20 \mathrm{~m}^{2}$. Se presentan plantas silvestres usadas como comestibles, forraje, combustible o leña, sombra y construcción. También esta zona se aprovecha para la quema y reciclaje de residuos orgánicos e inorgánicos de la vivienda.

El solar maya-ch'ol presenta dos subsistemas de producción, uno animal y otro vegetal. El subsistema animal está compuesto por la cría de animales domésticos, las aves se encuentran libres en el lugar y son usadas para la obtención de huevo y para el consumo de su carne en determinados eventos familiares. Además, algunas familias crían pollos de engorda para la venta de su carne, en un corral pequeño, generalmente de $2 \times 2 \mathrm{~m}$. También la crianza de cerdos principalmente para la venta.

El subsistema vegetal; está compuesto por la diversidad de especies de plantas cultivadas y silvestres, que presentan varias formas de aprovechamiento. En las zonas del traspatio y hortaliza se presentan principalmente especies alimenticias, conformadas por árboles frutales, arbustos y hierbas aprovechadas por sus tallos y hojas.

\section{Etnobotánica del solar maya-ch'ol del Valle del Tulijá, Chiapas}

En el solar maya-ch'ol de las comunidades del Valle del Tulijá, se registraron 131 especies de plantas útiles, pertenecientes a 58 familias botánicas. Las familias con mayor representación fueron Fabaceae (11 especies), Malvaceae y Rutaceae (8 especies cada una), Asteraceae, Myrtaceae y Solanaceae (5 especies cada una), Euphorbiaceae, y Apocynaceae (4 especies cada una). De todas las especies destaca el registro de 65 nombres en lengua ch'ol. Las especies con mayor valor de uso fueron aquellas de las que se aprovecha la misma planta para cubrir varias necesidades, de estas las más representativas con mayor valor de uso (IVUs) fueron Gliricidia sepium (Jacq.) Steud. (Fabaceae) (0.35), seguida de Mangifera indica L. (Anacardiaceae), Parmentiera aculata (Kunth) (Bignoniaceae) (cada una 0.30) y Erythrina folkersii Krukoff \& Moldenke (Fabaceae) (0.25) (Tabla 1).

Tabla 1: Lista de especies de plantas útiles del solar maya-ch'ol en las comunidades del Valle del Tulijá. Categorías antropocéntricas (Arte: artesanía, CeVi: cercas vivas, Cere: ceremoniales, Colo: colorantes, Comb: combustible, Comr: comercial, Come: comestible, Cosm: cosméticos, Esti: estimulante, Forr: forrajera, GoLa: gomas y látex, InAg: instrumentos de trabajo agrícola, Jugu: juguetes, MaCo: material de construcción, Med: medicina, Orna: ornamental, SeAm: servicio ambiental, Somb: sombra, UtDo: utensilios domésticos); Parte utilizada (H: hoja, FR: fruto, T: toda la planta, RA: ramas, TR: tronco, TA: tallo, SM: semilla, FL: flor, CO: corteza, RZ: raíz, EX: exudados, IN: inflorescencia, ES: espinas); Índice de Valor de Uso (IVUs).

\begin{tabular}{|c|c|c|c|c|c|}
\hline FAMILIA/especie & $\begin{array}{l}\text { Nombre } \\
\text { común/ch'ol }\end{array}$ & Usos & $\begin{array}{l}\text { Parte } \\
\text { utilizada }\end{array}$ & $I V U s$ & Datos de colección \\
\hline \multicolumn{6}{|l|}{ ACANTHACEAE } \\
\hline Justicia inaequalis Benth. & muites & Med, Orna & $\mathrm{T}$ & 0.1 & P. Ubiergo et al. 662 \\
\hline Ruellia simplex $\mathrm{C}$. Wright & petunia & Orna & FL & 0.05 & $\begin{array}{l}\text { P. Ubiergo y C. } \\
\text { Sánchez } 673\end{array}$ \\
\hline \multicolumn{6}{|l|}{ AMARANTHACEAE } \\
\hline Celosia argentea L. & & Orna & FL & 0.05 & P. Ubiergo et al. 607 \\
\hline Celosia cristata $\mathrm{L}$. & cresta de gallo & Orna & FL, T & 0.05 & $\begin{array}{l}\text { P. Ubiergo y C. } \\
\text { Sánchez } 608\end{array}$ \\
\hline \multicolumn{6}{|l|}{ AMARYLLIDACEAE } \\
\hline Allium fistulosum L. & cebollín/werux & Come, Med & TA, H & 0.1 & $\begin{array}{l}\text { A. Arcos y P. Ubiergo } \\
10\end{array}$ \\
\hline $\begin{array}{l}\text { Hippeastrum puniceum (Lam.) } \\
\text { Voss }\end{array}$ & azucena roja & Orna & FL & 0.05 & $\begin{array}{l}\text { P. Ubiergo y C. } \\
\text { Sánchez } 676\end{array}$ \\
\hline $\begin{array}{l}\text { ANACARDIACEAE } \\
\text { Mangifera indica } \mathrm{L} .\end{array}$ & mango/mankoj & $\begin{array}{l}\text { Comb,Come } \\
\text { Forr, aCo, } \\
\text { Med, Somb }\end{array}$ & FR, TA,T & 0.3 & $\begin{array}{l}\text { Y. Guzmán y P. } \\
\text { Ubiergo } 006\end{array}$ \\
\hline
\end{tabular}




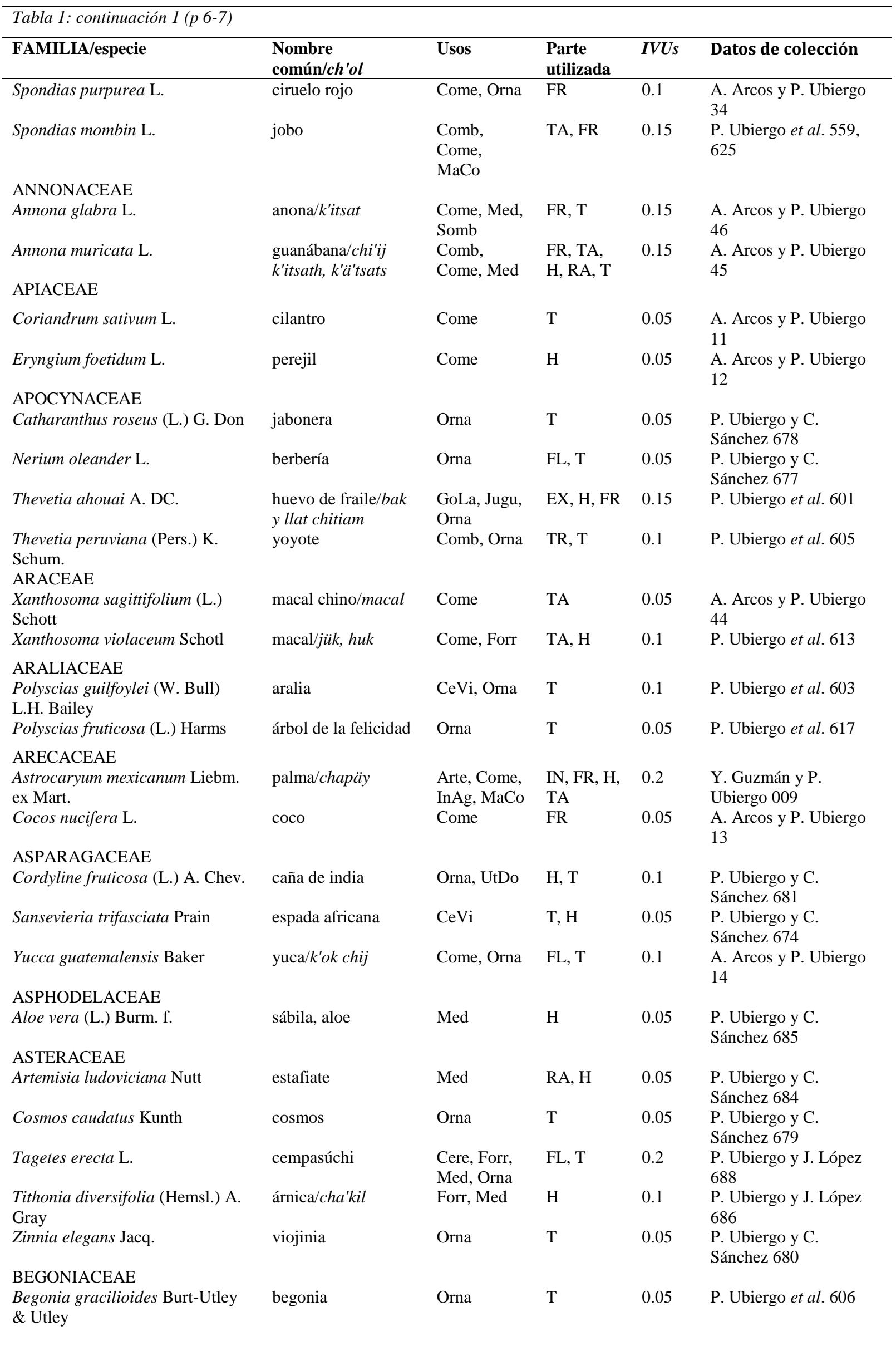




\begin{tabular}{|c|c|c|c|c|c|}
\hline FAMILIA/especie & $\begin{array}{l}\text { Nombre } \\
\text { común/ch'ol }\end{array}$ & Usos & $\begin{array}{l}\text { Parte } \\
\text { utilizada }\end{array}$ & IVUs & Datos de colección \\
\hline \multicolumn{6}{|l|}{ BIGNONIACEAE } \\
\hline Crescentia cujete $\mathrm{L}$. & morro/ty'e tsimá & Med, UtDo & FR & 0.1 & $\begin{array}{l}\text { P. Ubiergo y C. } \\
\text { Sánchez } 682\end{array}$ \\
\hline $\begin{array}{l}\text { Parmentiera aculata (Kunth) } \\
\text { Seem. }\end{array}$ & $\begin{array}{l}\text { cuajilote/chachib, } \\
\text { chichup, xchächäb }\end{array}$ & $\begin{array}{l}\text { Comb, } \\
\text { Come, Forr, } \\
\text { Med, Orna, } \\
\text { UtDo }\end{array}$ & FR, TA,T & 0.3 & P. Ubiergo et al. 618 \\
\hline \multicolumn{6}{|l|}{ BIXACEAE } \\
\hline Bixa orellana $\mathrm{L}$. & achiote & $\begin{array}{l}\text { CeVi, } \\
\text { Come, Med, } \\
\text { Somb }\end{array}$ & SM, T & 0.2 & P. Ubiergo 548 \\
\hline \multicolumn{6}{|l|}{ BORAGINACEAE } \\
\hline $\begin{array}{l}\text { Cordia alliodora (Ruiz \& Pav.) } \\
\text { Oken }\end{array}$ & $\begin{array}{l}\text { bojon/suijti'a, } \\
\text { suytya }\end{array}$ & $\begin{array}{l}\mathrm{CeVi} \\
\mathrm{MaCo} \\
\mathrm{SeAm} \\
\text { Somb }\end{array}$ & TA, T & 0.2 & $\begin{array}{l}\text { Y. Guzmán y P. } \\
\text { Ubiergo } 005\end{array}$ \\
\hline \multicolumn{6}{|l|}{ BROMELIACEAE } \\
\hline $\begin{array}{l}\text { Aechmea magdalenae (André) } \\
\text { André ex Baker }\end{array}$ & pita & $\mathrm{CeVi}$ & $\mathrm{T}$ & 0.05 & $\begin{array}{l}\text { P. Ubiergo y C. } \\
\text { Sánchez } 675\end{array}$ \\
\hline Ananas comosus (L.) Merr. & piña & $\begin{array}{l}\text { Come, } \\
\text { Comr, Med, } \\
\text { SeAm }\end{array}$ & $\mathrm{FR}, \mathrm{T}$ & 0.2 & $\begin{array}{l}\text { A. Arcos y P. Ubiergo } \\
15\end{array}$ \\
\hline \multicolumn{6}{|l|}{ BURSERACEAE } \\
\hline Bursera simaruba (L.) Sarg. & $\begin{array}{l}\text { palo mulato/chachä, } \\
\text { chakchí, châkaj }\end{array}$ & $\begin{array}{l}\text { CeVi, } \\
\text { MaCo, Med, } \\
\text { Somb }\end{array}$ & $\begin{array}{l}\text { TA, CO, } \\
\mathrm{T}\end{array}$ & 0.2 & $\begin{array}{l}\text { Y. Guzmán y P. } \\
\text { Ubiergo } 001\end{array}$ \\
\hline \multicolumn{6}{|l|}{ BUXACEAE } \\
\hline Buxus sempervirens L. & boj variegado & Orna & $\mathrm{T}$ & 0.05 & $\begin{array}{l}\text { P. Ubiergo y C. } \\
\text { Sánchez } 683\end{array}$ \\
\hline \multicolumn{6}{|l|}{ CACTACEAE } \\
\hline $\begin{array}{l}\text { Epiphyllum pumilum } \text { Britton \& } \\
\text { Rose }\end{array}$ & & Orna & TA & 0.05 & P. Ubiergo et al. 670 \\
\hline Hylocereus undatus (Haw.) & pitaya & Come & FR & 0.05 & A. Arcos y P. Ubiergo \\
\hline Britton \& Rose & & & & & 16 \\
\hline Opuntia ficus-indica (L.) Mill. & nopal & $\begin{array}{l}\text { Come, Med, } \\
\text { Orna }\end{array}$ & FR, TA, & 0.15 & P. Ubiergo et al. 538 \\
\hline \multicolumn{6}{|l|}{ CARICACEAE } \\
\hline Carica papaya $\mathrm{L}$. & $\begin{array}{l}\text { papaya silvestre/ } \\
\text { uchuñtye }^{\prime}\end{array}$ & Come, Med & FR, RA & 0.1 & $\begin{array}{l}\text { A. Arcos y P. Ubiergo } \\
18\end{array}$ \\
\hline \multicolumn{6}{|l|}{ CHENOPODIACEAE } \\
\hline Chenopodium ambrosioides $\mathrm{L}$. & epazote/pazoñthej & $\begin{array}{l}\text { Cere, Come, } \\
\text { Med }\end{array}$ & $\mathrm{H}$ & 0.15 & $\begin{array}{l}\text { A. Arcos y P. Ubiergo } \\
17\end{array}$ \\
\hline \multicolumn{6}{|l|}{ COMBRETACEAE } \\
\hline Terminalia catappa $\mathrm{L}$. & almendrón & $\begin{array}{l}\text { Come, } \\
\text { Orna, Somb }\end{array}$ & $\mathrm{T}, \mathrm{SM}$ & 0.15 & $\begin{array}{l}\text { A. Arcos y P. Ubiergo } \\
36\end{array}$ \\
\hline \multicolumn{6}{|l|}{ COMMELINACEAE } \\
\hline Tradescantia spathacea Sw. & $\begin{array}{l}\text { maguey } \\
\text { morado/chächäk } \\
\text { chij }\end{array}$ & Med, Orna & $\mathrm{H}$ & 0.1 & $\begin{array}{l}\text { P. Ubiergo y C. } \\
\text { Sánchez } 689\end{array}$ \\
\hline $\begin{array}{l}\text { CONVOLVULACEAE } \\
\text { Ipomoea carnea Jacq. }\end{array}$ & campanilla & Orna & $\mathrm{T}, \mathrm{FL}$ & 0.05 & $\begin{array}{l}\text { P. Ubiergo y C. } \\
\text { Sánchez } 694\end{array}$ \\
\hline Quamoclit vulgaris Choisy & mañanitas & Orna & $\mathrm{T}$ & 0.05 & P. Ubiergo et al. 609 \\
\hline $\begin{array}{l}\text { CRASSULACEAE } \\
\text { Bryophyllum daigremontianum } \\
\text { (Raym.-Hamet \& H. Perrier) A. } \\
\text { Berger }\end{array}$ & sanatodo & Med, Orna & $\mathrm{H}$ & 0.1 & $\begin{array}{l}\text { P. Ubiergo y C. } \\
\text { Sánchez } 691\end{array}$ \\
\hline $\begin{array}{l}\text { Bryophyllum pinnatum (Lam.) } \\
\text { Oken }\end{array}$ & 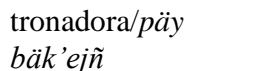 & Med, Orna & $\mathrm{H}, \mathrm{EX}$ & 0.1 & $\begin{array}{l}\text { P. Ubiergo y C. } \\
\text { Sánchez } 692\end{array}$ \\
\hline Sedum morganianum E. Walther & cola de borrego & Med, Orna & $\mathrm{T}$ & 0.1 & $\begin{array}{l}\text { P. Ubiergo y C. } \\
\text { Sánchez } 694\end{array}$ \\
\hline $\begin{array}{l}\text { CUCURBITACEAE } \\
\text { Sechium edule (Jacq.) Sw. }\end{array}$ & $\begin{array}{l}\text { chayote/ } \tilde{n} i j u^{\prime} k \text {, } \\
\tilde{n} i i u^{\prime} k\end{array}$ & Come & FR, H & 0.05 & $\begin{array}{l}\text { A. Arcos y P. Ubiergo } \\
33\end{array}$ \\
\hline
\end{tabular}


Ubiergo-Corvalán et al. El solar Maya-Ch’ol y sus saberes etnobotánicos em comunidades al Norte de Chiapas, México. Ethnoscientia 4, 2019. DOI 10.22276/ethnoscientia.v4i1.217

Tabla 1: continuación 3 (p.8-9)

\begin{tabular}{|c|c|c|c|c|c|}
\hline FAMILIA/especie & $\begin{array}{l}\text { Nombre } \\
\text { común/ch'ol }\end{array}$ & Usos & $\begin{array}{l}\text { Parte } \\
\text { utilizada }\end{array}$ & IVUs & Datos de colección \\
\hline \multicolumn{6}{|l|}{ EQUISETACEAE } \\
\hline Equisetum arvense $\mathrm{L}$. & cola de caballo & Med & $\mathrm{T}$ & 0.05 & $\begin{array}{l}\text { P. Ubiergo y J. López } \\
687\end{array}$ \\
\hline \multicolumn{6}{|l|}{ EUPHORBIACEAE } \\
\hline Acalypha wilkesiana Müll. Arg. & acalifa & $\mathrm{CeVi}$, Orna & $\mathrm{T}$ & 0.1 & P. Ubiergo et al. 604 \\
\hline $\begin{array}{l}\text { Codiaeum variegatum (L.) } \\
\text { Rumph. ex A. Juss. }\end{array}$ & croto & Orna & $\mathrm{T}$ & 0.05 & $\begin{array}{l}\text { P. Ubiergo y C. } \\
\text { Sánchez } 697\end{array}$ \\
\hline Euphorbia tithymaloides L. & zapatico de la reina & Orna & $\mathrm{T}$ & 0.05 & P. Ubiergo et al. 672 \\
\hline Jatropha curcas L. & piñón/bichruñtye & $\begin{array}{l}\mathrm{CeVi} \text {, } \\
\text { Come, Med }\end{array}$ & $\mathrm{H}, \mathrm{T}, \mathrm{FR}$ & 0.15 & $\begin{array}{l}\text { A. Arcos y P. Ubiergo } \\
19\end{array}$ \\
\hline \multicolumn{6}{|l|}{ FABACEAE } \\
\hline $\begin{array}{l}\text { Acaciella angustissima (Mill.) } \\
\text { Britton \& Rose }\end{array}$ & $\begin{array}{l}\text { carboncillo, } \\
\text { timbe/chekel, } \\
\text { chej'kel }\end{array}$ & Comb & TA & 0.05 & P. Ubiergo et al. 661 \\
\hline Cajanus cajan (L.) Huth & chícharo/tye bu'ul & Come & SM, FR & 0.05 & P. Ubiergo et al. 671 \\
\hline Canavalia ensiformis (L.) DC. & canavalia/permach & $\begin{array}{l}\text { Come, Med, } \\
\text { SeAm }\end{array}$ & $\mathrm{SM}, \mathrm{T}$ & 0.15 & $\begin{array}{l}\text { A. Arcos y P. Ubiergo } \\
43\end{array}$ \\
\hline $\begin{array}{l}\text { Crotalaria longirostrata Hook. \& } \\
\text { Arn. }\end{array}$ & chipilín & Come & $\mathrm{H}$ & 0.05 & $\begin{array}{l}\text { A. Arcos y P. Ubiergo } \\
42\end{array}$ \\
\hline $\begin{array}{l}\text { Erythrina folkersii Krukoff \& } \\
\text { Moldenke }\end{array}$ & $\begin{array}{l}\text { colorín/mötye, moj } \\
\text { thi'e }\end{array}$ & $\begin{array}{l}\mathrm{CeVi} \text {, } \\
\text { Comb, Forr, } \\
\text { Med, Somb }\end{array}$ & $\mathrm{H}, \mathrm{TA}, \mathrm{T}$ & 0.25 & P. Ubiergo et al. 600 \\
\hline $\begin{array}{l}\text { Dialium guianense (Aubl.) } \\
\text { Sandwith }\end{array}$ & $\begin{array}{l}\text { huapaque/wi'th, } \\
\text { wäch', wäch }\end{array}$ & $\begin{array}{l}\text { Comb, } \\
\text { Come, } \\
\text { MaCo, } \\
\text { Somb }\end{array}$ & TA, FR, T & 0.2 & $\begin{array}{l}\text { Y. Guzmán y P. } \\
\text { Ubiergo } 008\end{array}$ \\
\hline Gliricidia sepium (Jacq.) Steud. & cocoíte/xchântye' & $\begin{array}{l}\mathrm{CeVi} \text {, } \\
\text { Comb, } \\
\text { Come, Forr, } \\
\text { MaCo, Med, } \\
\text { Somb }\end{array}$ & $\mathrm{T}, \mathrm{FL}, \mathrm{T}$ & 0.35 & $\begin{array}{l}\text { Y. Guzmán y P. } \\
\text { Ubiergo } 002\end{array}$ \\
\hline $\begin{array}{l}\text { Inga inicuil Schltdl. \& Cham. ex } \\
\text { G. Don }\end{array}$ & $\begin{array}{l}\text { vaina de } \\
\text { monte/beats, } \\
\text { machitbitz }\end{array}$ & $\begin{array}{l}\text { Comb, } \\
\text { Come }\end{array}$ & FR, TA & 0.1 & $\begin{array}{l}\text { P. Ubiergo et al. } 540 \text {, } \\
599\end{array}$ \\
\hline Inga sapindoides Willd. & acocote/zeen bitys & $\begin{array}{l}\text { Comb, } \\
\text { Come }\end{array}$ & FR, TA & 0.1 & P. Ubiergo et al. 659 \\
\hline Phaseolus vulgaris $\mathrm{L}$. & $\begin{array}{l}\text { frijol, frijol negro, } \\
\text { frijol grande/ } \\
\text { wäcash bu'ul, } \\
\text { kaxlan bu'ul, pek } \\
\text { bu'ul, chu'bu'ul }\end{array}$ & Come & SM & 0.05 & P. Ubiergo et al. 547 \\
\hline Tamarindus indica $\mathrm{L}$. & tamarindo & $\begin{array}{l}\text { Comb, } \\
\text { Come, } \\
\text { MaCo, } \\
\text { Somb }\end{array}$ & TA, FR, T & 0.2 & $\begin{array}{l}\text { Y. Guzmán y P. } \\
\text { Ubiergo } 007\end{array}$ \\
\hline \multicolumn{6}{|l|}{ GESNERIACEAE } \\
\hline Episcia cupreata (Hook.) Hanst. & epicia/yäx bak & Med, Orna & $\mathrm{H}$ & 0.1 & $\begin{array}{l}\text { P. Ubiergo y C. } \\
\text { Sánchez } 698\end{array}$ \\
\hline \multicolumn{6}{|l|}{ LAMIACEAE } \\
\hline Ocimum campechianum Mill. & $\begin{array}{l}\text { albahaca } \\
\text { criolla/ixtyo'pimel }\end{array}$ & Come, Med & $\mathrm{H}$ & 0.1 & P. Ubiergo et al. 616 \\
\hline $\begin{array}{l}\text { Plectranthus amboinicus (Lour.) } \\
\text { Spreng. } \\
\text { LAURACEAE }\end{array}$ & oreganón & Med & $\mathrm{H}$ & 0.05 & $\begin{array}{l}\text { P. Ubiergo y C. } \\
\text { Sánchez } 693\end{array}$ \\
\hline Persea americana Mill. & aguacate/on & $\begin{array}{l}\text { Comb, } \\
\text { Come, Med, } \\
\text { Somb }\end{array}$ & FR, TA, T & 0.2 & $\begin{array}{l}\text { A. Arcos y P. Ubiergo } \\
37\end{array}$ \\
\hline Persea schiedeana Nees & chinín/koyoj & Come & FR & 0.05 & $\begin{array}{l}\text { A. Arcos y P. Ubiergo } \\
20\end{array}$ \\
\hline
\end{tabular}


Ubiergo-Corvalán et al. El solar Maya-Ch’ol y sus saberes etnobotánicos em comunidades al Norte de Chiapas, México. Ethnoscientia 4, 2019. DOI 10.22276/ethnoscientia.v4i1.217

Tabla 1: continuación 4 (p.9-10)

\begin{tabular}{|c|c|c|c|c|c|}
\hline FAMILIA/especie & $\begin{array}{l}\text { Nombre } \\
\text { común/ch'ol }\end{array}$ & Usos & $\begin{array}{l}\text { Parte } \\
\text { utilizada }\end{array}$ & IVUs & Datos de colección \\
\hline \multicolumn{6}{|l|}{ LOGANIACEAE } \\
\hline $\begin{array}{l}\text { Spigelia humboldtiana Cham. et } \\
\text { Schltdl. }\end{array}$ & $\begin{array}{l}\text { madre selva/ } x k^{\prime} o k \\
\text { pimel }\end{array}$ & Med & $\mathrm{T}$ & 0.05 & $\begin{array}{l}\text { P. Ubiergo y C. } \\
\text { Sánchez } 699\end{array}$ \\
\hline LORANTHACEAE & & & & & \\
\hline $\begin{array}{l}\text { Psittacanthus schiedeanus } \\
\text { (Schltdl. y Cham.) Blume. } \\
\text { MALPIGHIACEAE }\end{array}$ & muérdago/majañtye' & Med & $\mathrm{H}, \mathrm{TA}$ & 0.05 & $\begin{array}{l}\text { P. Ubiergo y C. } \\
\text { Sánchez } 703\end{array}$ \\
\hline Byrsonima crassifolia (L.) Kunth & nance/chi' & $\begin{array}{l}\text { Comb, } \\
\text { Come, Med, } \\
\text { Somb }\end{array}$ & $\begin{array}{l}\text { FR, TA, } \\
\text { T, CO, H }\end{array}$ & 0.20 & P. Ubiergo et al. 539 \\
\hline \multicolumn{2}{|l|}{ MALVACEAE } & Cosm, Orna & $\mathrm{T}$ & 0.1 & P. Ubiergo et al. 549 \\
\hline Hibiscus rosa-sinensis $\mathrm{L}$. & tulipán & $\begin{array}{l}\text { CeVi, Forr, } \\
\text { Orna, UtDo }\end{array}$ & $\mathrm{T}$ & 0.2 & P. Ubiergo et al. 638 \\
\hline Malachra capitata (L.) L. & malva/tsuts chikiñ & Med & $\mathrm{H}, \mathrm{RA}$ & 0.05 & P. Ubiergo et al. 623 \\
\hline Malvaviscus arboreus Cav. & $\begin{array}{l}\text { tulipán } \\
\text { silvestre/chächäk } \\
\text { nichim }\end{array}$ & $\begin{array}{l}\text { CeVi, Med, } \\
\text { Orna, UtDo }\end{array}$ & $\mathrm{H}, \mathrm{FL}$ & 0.2 & P. Ubiergo et al. 602 \\
\hline Pachira aquatica Aubl. & $\begin{array}{l}\text { zapote de } \\
\text { agua/xchu'ch }\end{array}$ & $\begin{array}{l}\text { Comb, } \\
\text { Come, } \\
\text { MaCo, } \\
\text { Somb }\end{array}$ & TA, T & 0.2 & $\begin{array}{l}\text { Y. Guzmán y P. } \\
\text { Ubiergo } 003\end{array}$ \\
\hline Sida rhombifolia L. & chichibe & Med & $\mathrm{T}$ & 0.05 & P. Ubiergo et al. 664 \\
\hline Theobroma cacao $\mathrm{L}$. & cacao/kâkâw & $\begin{array}{l}\text { Come, } \\
\text { SeAm }\end{array}$ & $\begin{array}{l}\text { FR, SM, } \\
\mathrm{H}\end{array}$ & 0.1 & $\begin{array}{l}\text { A. Arcos y P. Ubiergo } \\
38\end{array}$ \\
\hline Theobroma bicolor Bonpl. & $\begin{array}{l}\text { pataste/sâsâk } \\
\text { kâkâw, pataxte, } \\
\text { bajlumtye }\end{array}$ & Come & $\mathrm{FR}, \mathrm{SM}$ & 0.05 & P. Ubiergo et al. 550 \\
\hline \multicolumn{6}{|l|}{ MARANTACEAE } \\
\hline Maranta arundinacea $\mathrm{L}$. & azafrán/yopom & $\begin{array}{l}\text { Come, Forr, } \\
\text { Orna, UtDo }\end{array}$ & $\mathrm{H}, \mathrm{RZ}$ & 0.2 & P. Ubiergo et al. 582 \\
\hline \multicolumn{6}{|l|}{ MORACEAE } \\
\hline $\begin{array}{l}\text { Artocarpus altilis (Parkinson) } \\
\text { Fosberg }\end{array}$ & castaña & $\begin{array}{l}\text { Come, } \\
\text { Somb }\end{array}$ & $\mathrm{T}, \mathrm{FR}$ & 0.1 & $\begin{array}{l}\text { A. Arcos y P. Ubiergo } \\
21\end{array}$ \\
\hline Ficus benjamina $\mathrm{L}$. & ficus & Orna, Somb & TA, T & 0.1 & $\begin{array}{l}\text { Y. Guzmán y P. } \\
\text { Ubiergo } 011\end{array}$ \\
\hline \multicolumn{6}{|l|}{ MUNTINGIACEAE } \\
\hline Muntingia calabura $\mathrm{L}$. & capulín/kapuk & $\begin{array}{l}\text { CeVi, } \\
\text { Come, } \\
\text { Somb }\end{array}$ & $\mathrm{FR}, \mathrm{T}$ & 0.15 & $\begin{array}{l}\text { A. Arcos y P. Ubiergo } \\
39\end{array}$ \\
\hline \multicolumn{6}{|l|}{ MUSACEAE } \\
\hline Musa acuminata Colla & $\begin{array}{l}\text { plátano dulce/âlâ } \\
\text { tsaj-ja'as }\end{array}$ & Come & $\mathrm{FR}, \mathrm{H}$ & 0.05 & $\begin{array}{l}\text { A. Arcos y P. Ubiergo } \\
23\end{array}$ \\
\hline Musa balbisiana Colla & $\begin{array}{l}\text { plátano cuadrado, } \\
\text { plátano macho/ } \\
\text { pepech ja'as, } x \text { - } \\
\text { ichim ja'as }\end{array}$ & Come & $\mathrm{FR}, \mathrm{H}$ & 0.05 & $\begin{array}{l}\text { A. Arcos y P. Ubiergo } \\
24\end{array}$ \\
\hline Musa paradisiaca $\mathrm{L}$. & $\begin{array}{l}\text { plátano rojo, } \\
\text { plátano } \\
\text { guineo/châchâk } \\
\text { ja'as, âlâ ja'as } \\
\text { rubatan ja'as }\end{array}$ & Come, Forr & FR & 0.1 & $\begin{array}{l}\text { A. Arcos y P. Ubiergo } \\
22\end{array}$ \\
\hline \multicolumn{6}{|l|}{ MYRTACEAE } \\
\hline Eugenia acapulcensis Steud. & capulín/chikt, chityi & $\begin{array}{l}\text { Comb, } \\
\text { Come, Med }\end{array}$ & FR & 0.15 & $\begin{array}{l}\text { A. Arcos y P. Ubiergo } \\
41\end{array}$ \\
\hline Syzygium jambos (L.) Alston & pomarosa & $\begin{array}{l}\text { Comb, } \\
\text { Come, } \\
\text { Somb }\end{array}$ & TA, FR & 0.15 & $\begin{array}{l}\text { A. Arcos y P. Ubiergo } \\
25\end{array}$ \\
\hline $\begin{array}{l}\text { Syzygium malaccense (L.) Merr. } \\
\text { \& L.M. Perry }\end{array}$ & pomagas & $\begin{array}{l}\text { Comb, } \\
\text { Come, } \\
\text { Somb }\end{array}$ & TA, FR & 0.15 & $\begin{array}{l}\text { A. Arcos y P. Ubiergo } \\
26\end{array}$ \\
\hline Pimenta dioica (L.) Merr. & pimienta silvestre & $\begin{array}{l}\text { Comb, } \\
\text { Come, } \\
\text { Somb }\end{array}$ & FR, TA, T & 0.15 & $\begin{array}{l}\text { A. Arcos y P. Ubiergo } \\
40\end{array}$ \\
\hline
\end{tabular}


Ubiergo-Corvalán et al. El solar Maya-Ch’ol y sus saberes etnobotánicos em comunidades al Norte de Chiapas, México. Ethnoscientia 4, 2019. DOI 10.22276/ethnoscientia.v4i1.217

\begin{tabular}{|c|c|c|c|c|c|}
\hline \multicolumn{6}{|l|}{ Tablal: continuación 5(p.10-11) } \\
\hline FAMILIA/especie & $\begin{array}{l}\text { Nombre } \\
\text { común/ch'ol }\end{array}$ & Usos & $\begin{array}{l}\text { Parte } \\
\text { utilizada }\end{array}$ & $I V U s$ & Datos de colección \\
\hline Psidium guajava $\mathrm{L}$. & guayaba/pâtyaj & $\begin{array}{l}\text { Comb, } \\
\text { Come, Med, } \\
\text { Somb }\end{array}$ & TA, FR, H & 0.2 & P. Ubiergo et al. 541 \\
\hline $\begin{array}{l}\text { NYCTAGINACEAE } \\
\text { Bougainvillea spectabilis Willd. }\end{array}$ & bugambilia & Med, Orna & FL, H & 0.1 & P. Ubiergo 577 \\
\hline $\begin{array}{l}\text { OXALIDACEAE } \\
\text { Averrhoa carambola } \mathrm{L} \text {. }\end{array}$ & carambola & Come & FR & 0.05 & $\begin{array}{l}\text { A. Arcos y P. Ubiergo } \\
49\end{array}$ \\
\hline $\begin{array}{l}\text { PETIVERIACEAE } \\
\text { Petiveria alliacea } \mathrm{L} \text {. }\end{array}$ & zorrillo/pajây & Cere, Med & $\mathrm{RZ}, \mathrm{H}$ & 0.1 & $\begin{array}{l}\text { P. Ubiergo y C. } \\
\text { Sánchez } 700\end{array}$ \\
\hline $\begin{array}{l}\text { PIPERACEAE } \\
\text { Piper auritum Kunth }\end{array}$ & hoja santa/mömöy & $\begin{array}{l}\text { Come, Forr, } \\
\text { Med }\end{array}$ & $\begin{array}{l}\mathrm{H}, \mathrm{TA}, \\
\mathrm{RZ}\end{array}$ & 0.15 & P. Ubiergo et al. 621 \\
\hline $\begin{array}{l}\text { PLANTAGINACEAE } \\
\text { Plantago major } \mathrm{L} .\end{array}$ & yantén/yante & Med & $\mathrm{T}$ & 0.05 & $\begin{array}{l}\text { P. Ubiergo y C. } \\
\text { Sánchez } 695\end{array}$ \\
\hline $\begin{array}{l}\text { POACEAE } \\
\text { Cymbopogon citratus (DC.) Stapf }\end{array}$ & zacate limón & Med, Orna & $\mathrm{H}$ & 0.1 & $\begin{array}{l}\text { P. Ubiergo y C. } \\
\text { Sánchez } 702\end{array}$ \\
\hline $\begin{array}{l}\text { PORTULACACEAE } \\
\text { Portulaca grandiflora Hook. }\end{array}$ & mañanitas & Orna & $\mathrm{T}$ & 0.05 & P. Ubiergo et al. 608 \\
\hline $\begin{array}{l}\text { Portulaca oleracea } \mathrm{L} \text {. } \\
\text { ROSACEAE }\end{array}$ & verdolaga & Come, Orna & $\mathrm{T}$ & 0.1 & P. Ubiergo et al. 612 \\
\hline Rosa sp. & rosa & Cere, Orna & $\mathrm{T}$ & 0.1 & P. Ubiergo et al. 614 \\
\hline $\begin{array}{l}\text { RUBIACEAE } \\
\text { Arachnothryx } \\
\text { leucophylla (Kunth) Planch. }\end{array}$ & panamá rose & Orna & FL, $\mathrm{T}$ & 0.05 & $\begin{array}{l}\text { P. Ubiergo y C. } \\
\text { Sánchez } 701\end{array}$ \\
\hline Hamelia patens Jacq. & $\begin{array}{l}\text { coralillo/käntye, } \\
\text { xchäk tyaj tye' }\end{array}$ & Med, Orna & $\mathrm{H}$ & 0.1 & P. Ubiergo et al. 596 \\
\hline Morinda citrifolia $\mathrm{L}$. & noni/k'äñ tye' & $\begin{array}{l}\text { Come, Forr, } \\
\text { Med }\end{array}$ & $\mathrm{FR}, \mathrm{CO}$ & 0.15 & $\begin{array}{l}\text { A. Arcos y P. Ubiergo } \\
27\end{array}$ \\
\hline RUTACEAE & & & & & \\
\hline Citrus aurantium $\mathrm{L}$. & naranja agría, cajera & $\begin{array}{l}\text { Comb, } \\
\text { Come, } \\
\text { Somb }\end{array}$ & FR, TA,T & 0.15 & $\begin{array}{l}\text { A. Arcos y P. Ubiergo } \\
31\end{array}$ \\
\hline Citrus sinensis (L.) Osbeck & naranja & $\begin{array}{l}\text { Comb, } \\
\text { Come, } \\
\text { Somb }\end{array}$ & TR, FR, T & 0.15 & $\begin{array}{l}\text { A. Arcos y P. Ubiergo } \\
50\end{array}$ \\
\hline Citrus maxima (Burm.) Merr. & toronja/toronka & Come & FR & 0.05 & $\begin{array}{l}\text { A. Arcos y P. Ubiergo } \\
28\end{array}$ \\
\hline Citrus nobilis Lour. & limón mandarina & Come & FR & 0.05 & $\begin{array}{l}\text { A. Arcos y P. Ubiergo } \\
32\end{array}$ \\
\hline Citrus limetta Risso & lima & $\begin{array}{l}\text { Come, Med, } \\
\text { Somb }\end{array}$ & FR, T, FL & 0.15 & $\begin{array}{l}\text { A. Arcos y P. Ubiergo } \\
29\end{array}$ \\
\hline Citrus limon (L.) Osbeck & limón/chu'j limoñ & $\begin{array}{l}\text { Come, Med, } \\
\text { SeAm }\end{array}$ & $\mathrm{FR}, \mathrm{T}$ & 0.15 & $\begin{array}{l}\text { A. Arcos y P. Ubiergo } \\
30\end{array}$ \\
\hline Citrus reticulata Blanco & $\begin{array}{l}\text { mandarina/mantarin } \\
a\end{array}$ & Come, Forr & FR & 0.1 & $\begin{array}{l}\text { A. Arcos y P. Ubiergo } \\
35\end{array}$ \\
\hline Ruta graveolens $\mathrm{L}$. & ruda & Med & $\mathrm{T}$ & 0.05 & $\begin{array}{l}\text { P. Ubiergo y C. } \\
\text { Sánchez } 696\end{array}$ \\
\hline $\begin{array}{l}\text { SAPINDACEAE } \\
\text { Cupania glabra } \mathrm{Sw} \text {. }\end{array}$ & $\begin{array}{l}\text { quiebra } \\
\text { hacha/chäkchöm }\end{array}$ & Comb & TA & 0.05 & $\begin{array}{l}\text { Y. Guzmán y P. } \\
\text { Ubiergo } 010\end{array}$ \\
\hline Melicoccus bijugatus Jacq. & guaya & Come & FR & 0.05 & $\begin{array}{l}\text { A. Arcos y P. Ubiergo } \\
47\end{array}$ \\
\hline $\begin{array}{l}\text { SAPOTACEAE } \\
\text { Chrysophyllum } \\
\text { mexicanum Brandegee ex Standl. }\end{array}$ & caimito/tyulum & $\begin{array}{l}\text { Come, } \\
\text { UtDo }\end{array}$ & FR, T & 0.1 & $\begin{array}{l}\text { A. Arcos y P. Ubiergo } \\
48\end{array}$ \\
\hline
\end{tabular}


Ubiergo-Corvalán et al. El solar Maya-Ch’ol y sus saberes etnobotánicos em comunidades al Norte de Chiapas, México. Ethnoscientia 4, 2019. DOI 10.22276/ethnoscientia.v4i1.217

Tabla 1: continuación

\begin{tabular}{|c|c|c|c|c|c|}
\hline FAMILIA/especie & $\begin{array}{l}\text { Nombre } \\
\text { común/ch'ol }\end{array}$ & Usos & $\begin{array}{l}\text { Parte } \\
\text { utilizada }\end{array}$ & IVUs & Datos de colección \\
\hline $\begin{array}{l}\text { Pouteria sapota (Jacq.) H.E. } \\
\text { Moore \& Stearn }\end{array}$ & zapote mamey & $\begin{array}{l}\text { Come, } \\
\text { MaCo, } \\
\text { Somb }\end{array}$ & TA, FR, T & 0.15 & $\begin{array}{l}\text { Y. Guzmán y P. } \\
\text { Ubiergo } 004\end{array}$ \\
\hline \multicolumn{6}{|l|}{ SOLANACEAE } \\
\hline Capsicum аппиит $\mathrm{L}$. & $\begin{array}{l}\text { chile pico de } \\
\text { paloma, } \\
\text { cimarrón/simaroich, } \\
\text { ich }\end{array}$ & $\begin{array}{l}\text { Cere, Come, } \\
\text { Med }\end{array}$ & FR & 0.15 & P. Ubiergo et al. 611 \\
\hline Lycopersicon esculentum Mill. & tomate/loth'is coyaj & Come & FR & 0.05 & P. Ubiergo et al. 546 \\
\hline Nicotiana tabacum $\mathrm{L}$. & tabaco & $\begin{array}{l}\text { Cere, Esti, } \\
\text { Med }\end{array}$ & $\mathrm{H}$ & 0.15 & P. Ubiergo et al. 636 \\
\hline Physalis pubescens $\mathrm{L}$. & $\begin{array}{l}\text { tomatillo } \\
\text { verde/chuk'uy }\end{array}$ & Come & FR & 0.05 & P. Ubiergo et al. 645 \\
\hline $\begin{array}{l}\text { Witheringia meiantha (Donn. } \\
\text { Sm.) Hunz. } \\
\text { VERBENACEAE }\end{array}$ & $\begin{array}{l}\text { ashante/ashantye, } \\
\text { ashänthe }\end{array}$ & Come, Med & $\mathrm{H}$ & 0.1 & P. Ubiergo et al. 610 \\
\hline Verbena litoralis Kunth & $\begin{array}{l}\text { verbena/xwich'ak'ac } \\
h\end{array}$ & Med & RA & 0.05 & P. Ubiergo et al. sn \\
\hline \multicolumn{6}{|l|}{ ZINGIBERACEAE } \\
\hline Zingiber officinale Roscoe & $\begin{array}{l}\text { jengibre criollo/san } \\
\text { sibre }\end{array}$ & Come, Med & TA & 0.1 & $\begin{array}{l}\text { P. Ubiergo et al. } 544 \text {, } \\
615\end{array}$ \\
\hline Renealmia alpinia (Rottb.) Maas & $\begin{array}{l}\text { cargamomo/wäum, } \\
\text { w'aum ch'e }\end{array}$ & $\begin{array}{l}\text { Colo, Come, } \\
\text { UtDo }\end{array}$ & $\begin{array}{l}\mathrm{FR}, \mathrm{H}, \\
\mathrm{SM}\end{array}$ & 0.15 & $\begin{array}{l}\text { P. Ubiergo et al. 553, } \\
597\end{array}$ \\
\hline
\end{tabular}

Del uso de las plantas registradas se clasificaron 19 categorías antropocéntricas, la descripción de cada una se realizó con la información suministrada por los informantes. Las categorías con mayor representación fueron: comestible (70 especies), medicinal (53 especies), ornamental (46 especie), sombra (24 especies), combustible ( 23 especies), cercas vivas y forraje (13 especies cada una) y material para la construcción (10 especies), las demás categorías con menos de ocho especies fueron poco frecuente (Figura 2).

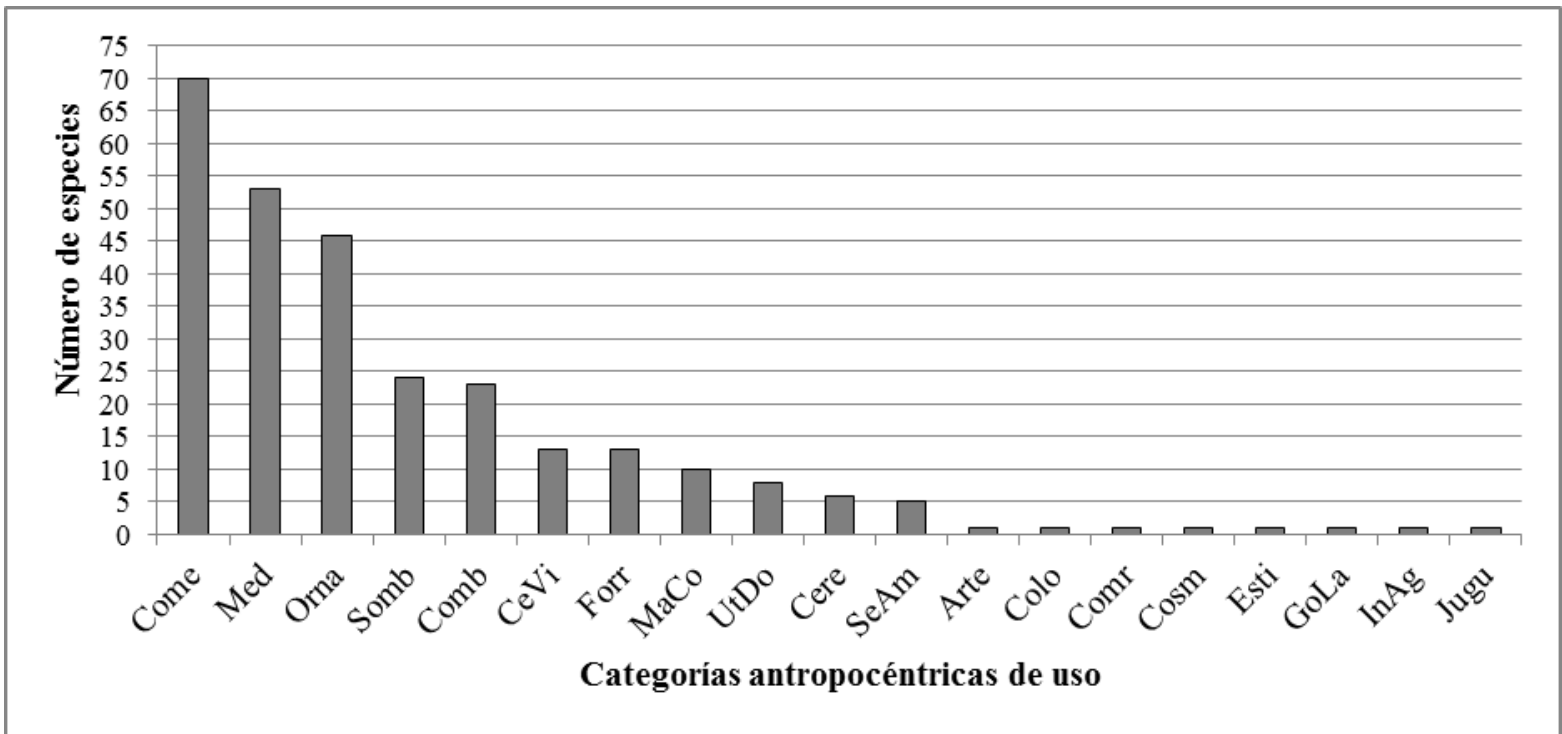

Figura 2: Categorías antropocéntricas de uso de las plantas en el solar maya-ch'ol de las comunidades del Valle del Tulijá. (Arte: artesanía, CeVi: cercas vivas, Cere: ceremoniales, Colo: colorantes, Comb: combustible, Comr: comercial, Come: comestible, Cosm: cosméticos, Esti: estimulante, Forr: forrajera, GoLa: gomas y látex, InAg: instrumentos de trabajo agrícola, Jugu: juguetes, MaCo: material de construcción, Med: medicina, Orna: ornamental, SeAm: servicio ambiental, Somb: sombra, UtDo: utensilios domésticos).

El biotipo más frecuente entre las plantas útiles del solar fueron los árboles (39\%) con 145 formas de uso; seguido de las hierbas (34\%) con 67 formas de uso; y los arbustos (20\%) con 51 formas de usos, mientras que otros biotipos (trepadora, roseta y epífitas) fueron menos comunes (Figura 3). 


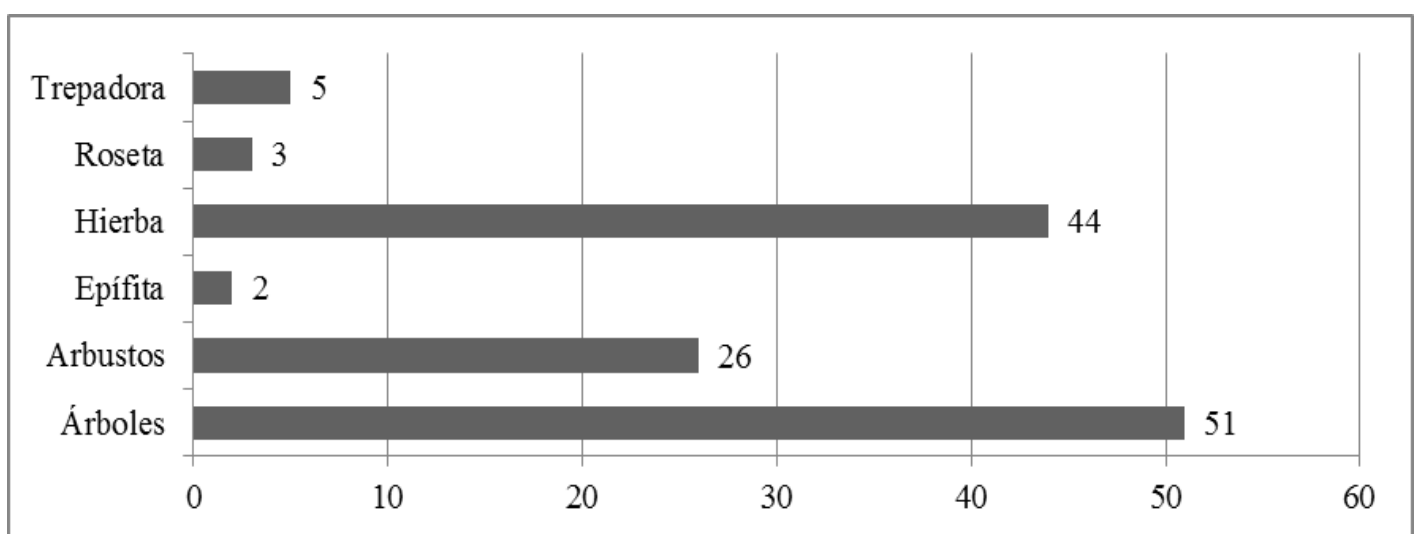

Figura 3: Biotipos de las plantas útiles del solar maya-ch 'ol de las comunidades del Valle del Tulijá, Chiapas.

La relación entre el biotipo y las categorías de uso refleja que los árboles son mayormente utilizados como comestible (42 especies), sombra (24 especies), combustible (23 especies), medicinal (19 especies) y material para la construcción (10 especies); seguido de las hierbas empleadas como medicinal (22 especies), ornamental (16 especies) y comestible (13 especies); y los arbustos representados en uso ornamental (20 especies), medicinal (9 especies) y comestible (8 especies) (Figura 4).

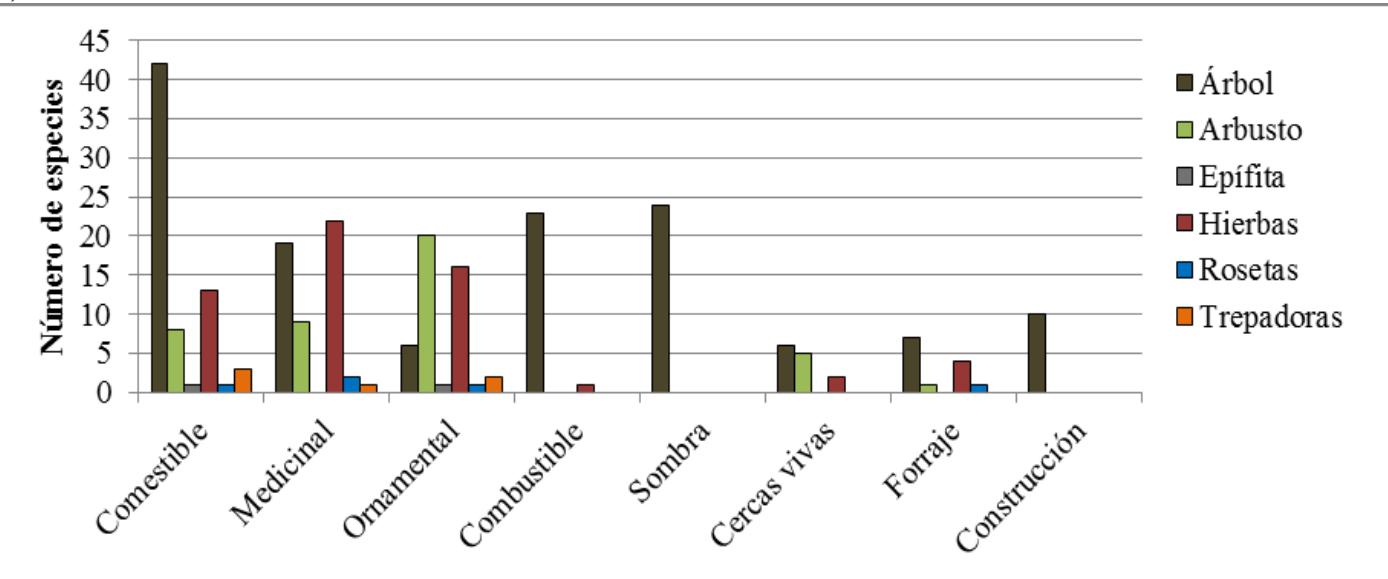

Figura 4: Relación entre la categoría de uso y el biotipo de las plantas del solar maya-ch'ol en las comunidades del Valle del Tulijá, Chiapas.

La estructura de la planta más utilizada en el solar es el fruto (52 especies) comúnmente usado como comestible, seguido de la hoja (39 especies) generalmente para la preparación de medicamentos, toda la planta (37 especies) la mayoría utilizada como ornamental y el tallo (33 especies) usado con frecuencia para material de construcción y combustible (Figura 5).

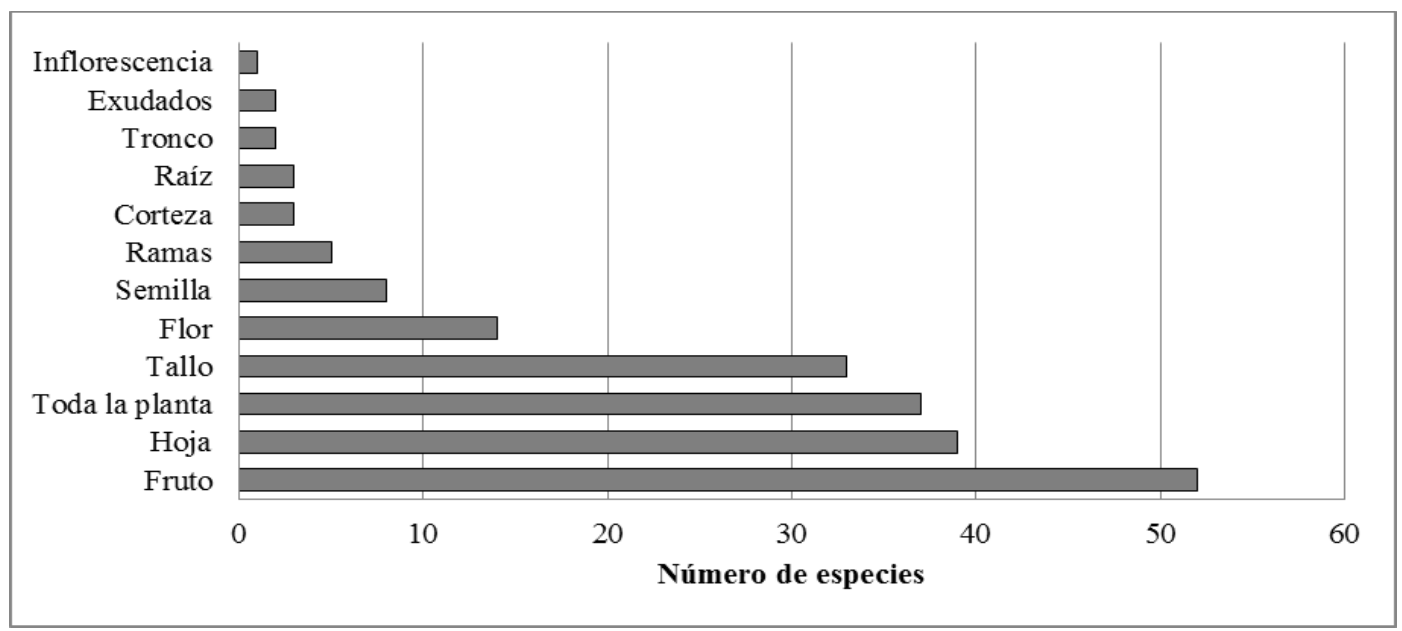

Figura 5: Estructuras de las plantas utilizadas en el solar maya ch'ol de las comunidades del Valle del Tulijá, Chiapas, México. 


\section{DISCUSIÓN}

\section{El solar maya-ch'ol en el Valle del Tulijá, Chiapas}

Las tres localidades estudiadas en esta investigación corresponden a la región del Valle del Tulijá, ubicadas entre $50 \mathrm{~m}$ y $1300 \mathrm{~m}$ de distancia del río Tulijá, el cual según sus habitantes, representa un recurso valioso para sus sistemas de vida y ha sido elemento importante en la definición del mismo; aspectos que fueron considerados por Andrade y Mejía (2015), donde señalan que el territorio puede ser considerado un elemento relevante para la percepción y formas de organización de los grupos originarios; no obstante, estos autores detallan como fue el proceso de transformación que ha ocurrido en el territorio ch'ol, como cambios de uso de suelo, aceptación de la religión cristiana, inclusión de corporaciones extranjeras, actividades turísticas, y actuales políticas públicas.

La milpa y el solar representan los agroecosistemas de mayor importancia en la unidad de producción familiar de estas comunidades maya-ch'ol, sus diversos componentes contribuyen principalmente en los procesos de autoabastecimiento y conservación de especies de plantas útiles, lo que ha sido señalado por Aguirre-Ruíz et al. (2013) y Aguilar (2014), también han señalado al espacio del solar, como una zona de convivencia, trabajo y organización familiar; aspectos que han sido reconocidos para los huertos familiares de Chiapas (RODRÍGUEZ-GALVÁN, 2011; CABALLEROROQUE et al., 2018).

El solar maya-ch'ol presentó cuatro espacios zonificados destinados para diversas formas de aprovechamiento y beneficio familiar, similar a lo reportado por Contreras-Cortés et al. (2013) para los huertos de los lacandones en Nahá, Chiapas, donde también organizan diversos espacios para el autoconsumo, venta, y uso de diversas especies de plantas alimenticias y medicinales. Asimismo, la presencia de algunas especies de origen silvestre evidencia la posibilidad de que se presenten áreas de domesticación en el solar, aspecto que se ha indicado en diversos estudios relacionados con los factores sociales, manejo agrícola y los procesos de domesticación (CASAS et al., 2014; CABALLERO-ROQUE et al.; 2018).

En el solar la familia se encarga de criar animales de traspatio, con la finalidad de autoabastecimiento, producción comercial y/o acompañamiento; lo que coincide con Lerner et al. (2009) y Contreras-Cortés et al. (2013) para las comunidades del norte de Chiapas, quienes destacan la presencia de aves (gallinas, guajolotes, patos) y cerdos como los grupos de animales mejor representados. Lerner et al. (2009) indica para el caso de las gallinas, el valor que le dan a los especímenes de genética local, dado que son más abundantes, aseguran aporte de huevo y carne a la dieta familiar, además requieren de una menor inversión monetaria en alimento que los cerdos.

La vivienda es el espacio de convivencia y refugio, donde la familia realiza las actividades domésticas, aspecto afianzado por Torres (2010) y Mariaca (2012), quienes indican que este sitio constituye el espacio donde se genera y comparte conocimiento para el desarrollo de diversas actividades familiares. Los materiales de construcción de la vivienda han variado debido al cambio del uso de recursos, y la vinculación con programas de gobierno; sin embargo, el uso de la madera obtenida de las especies como Dialium guianense (Aubl.) Sandwith (Fabaceae), Spondias mombin L. (Anacardiaceae) y Pouteria sapota (Jacq.) H.E. Moore \& Stearn (Sapotaceae) presentes en el traspatio, continúan siendo un elemento indispensable en la construcción; para los pueblos mayas de Yucatán, Hernández (2010) señala también la distribución similar en el solar, y diferencias en el uso de diversos materiales de construcción.

Según los resultados obtenidos en las encuestas, la familia ch'ol se compone de un núcleo conyugal y de familias colectivas, lo que es común en diversos grupos indígenas mayas (MARIACA, 2012). El padre jefe de familia es el encargado principal del manejo tradicional de los agroecosistemas; sin embargo, en el solar el rol principal en las actividades del manejo del traspatio está representado por la madre jefa de familia, lo que concuerda con Arcos (2013), quien señala que la mujer ch'ol contribuye en la crianza de animales de corral. A su vez, esta información ha sido reconocida para varios estudios dedicados al traspatio en otras regiones indígenas de Chiapas y Latinoamérica (RODRÍGUEZ-GALVÁN, 2011; CONTRERAS-CORTÉS et al., 2013; QUINTERO et al., 2015; MENDOZA, 2015).

Los subsistemas productivos en el solar están destinados para autoabastecer a la familia, muy poca producción tiene una finalidad comercial. Aunque la forma de ingreso económico, principalmente es el trabajo agropecuario, como también se ha mencionado por Arcos (2013) y Aguilar (2014), se presentaron pocas familias con negocios propios, u organizadas para la comercialización de su producción. Según Aguilar (2014), este territorio está definido por la cultura tradicional de cultivo, destinada al autoconsumo y comercialización regional; sin embargo existe baja 
presencia de organizaciones sociales y productivas constituidas legalmente para la elaboración, gestión y puesta en marcha de proyectos productivos del sector agropecuario.

\section{Etnobotánica del solar maya-ch'ol en el Valle del Tulijá, Chiapas}

En relación con los saberes etnobotánicos del solar de las comunidades estudiadas; se reportaron 131 especies útiles, similar a lo registrado por Lerner et al. (2009) donde reporta 142 especies en su estudio sobre huertos en una comunidad ch'ol del municipio Salto de Agua, aunque su estudio fue orientado principalmente a las especies comestibles, registra datos importantes en cuanto a la diversidad de especies en el huerto familiar y la milpa. De igual manera, en un estudio previo, Vogl et al. (2002), reportaron 241 especies en huertos de tres comunidades tzeltal, ch'ol y mestiza del municipio de Palenque, Chiapas; estos autores señalaron que la comunidad ch'ol presenta mayor diversidad con 202 especies útiles, destaca que los agricultores de esta etnia reconocen más sus prácticas culturales y agrícolas que los agricultores tzeltales y mestizos en esa región.

Para el estado de Chiapas, se presentan datos mayores a lo registrado en este estudio, así Mariaca (2012) señala 426 especies útiles presentes en huertos familiares, aunque se presenta un extenso listado florístico donde incluye estudios en 36 localidades del estado, aún se presentan vacíos en algunas regiones y comunidades de pueblos indígenas, tal como lo señala actualmente CaballeroRoque et al. (2018); no obstante, los datos específicos para las comunidades indígenas indicados por este último autor, presentan rangos de diversidad de especies útiles similares a los reportados por este estudio.

En otras culturas mayas de México se reportan resultados mayores en cuanto al número de especies; así Neulinger et al. (2013), en un estudio de huertos de Campeche señalaron 310 especies de plantas usadas por comunidades mayas y mestiza, de las cuales 185 son usadas por los mayas, de estas 35 especies fueron registradas exclusivamente en los huertos mayas. Posiblemente, la integración de comunidades mestizas haya incorporado el uso de especies provenientes de otras regiones, lo que puede aumentar el conocimiento del uso de plantas.

Las familias botánicas más representativas en función a su número de especies son Fabaceae, Asteraceae y Malvaceae, lo que concuerda con Toledo et al. (1995), en la recopilación de estudios etnobotánicos de los grupos indígenas de la selva tropical, estudiados hasta ese momento en México; incluyen para el estado de Chiapas sólo investigaciones del pueblo Zoque y Lacandón. De igual manera, Levy et al. (2002) en un estudio etnobotánico para la comunidad Lacandona de Lacanhá, Chiapas, señalaron a las Fabaceae, Rubiaceae y Asteraceae como las familias más importantes de acuerdo al número de especies útiles en la flora espontánea de ese territorio.

Cabe mencionar que estas familias son parte importante del tipo de vegetación de selva baja perennifolia que predomina en la región, particularmente la familia Fabaceae como la más representativa tanto en número de especies y en la mayoría de formas biológicas, como se ha señalado por diversos autores (TOLEDO et al., 1995; LEVY et al., 2002; RZEDOWSKI, 2006; LÓPEZ y VALDEZ, 2011; AGUILAR, 2014).

Las especies que presentaron valores significativos de $I V U$, son aquellas que se aprovechan para diversos usos, y se utilizan, en general varias estructuras de la planta para cubrir diversas necesidades, destaca que todas son especies cultivadas en el solar, la mayoría son árboles y conocidas por la comunidad con sus nombres en castellano y lengua ch'ol. Asimismo, algunos autores han señalado la relación del valor de uso con el ambiente de procedencia de las especies; de tal manera que Sol et al. (2016), indica en su trabajo que la especie con el máximo valor de uso se debe a la adaptación que esta presenta con el ecosistema de la localidad de estudio y por la diversidad de formas de usos que presenta. Otros autores, Hurtado y Moraes (2010) también señalan en dos localidades en Bolivia que los valores más significativos del $I V U$ se encuentran en el área de los cultivos, indicando que es un hábitat de importancia para la comunidad.

La planta llamada cocohíte Gliricidia sepium (Jacq.) Steud. (Fabaceae), representa la especie con mayor valor de uso del solar, esto significa que es la especie conocida por las comunidades que presenta mayor número de formas de uso en beneficio de las familias. De igual manera, Mariaca (2012) ha señalado en su listado de plantas para el estado, al cocohíte como la especie que presenta hasta 14 categorías de uso; así como por diferentes autores para otros estados del sur de México (GUILLERMO, 2013; NEULINGER et al., 2013).

Se mencionó un porcentaje significativo de nombre de plantas en lengua ch'ol, similar a lo registrado también por Lerner et al. (2009), en un listado de plantas con nombres en ch'ol por especie. Aulie y Aulie (2009) en el vocabulario ch'ol de Tumbalá, Chiapas, registran 127 nombres de especies 
de plantas útiles, al comparar con los resultados de este estudio, se observa que sólo 37 especies coinciden con los nombres comunes y científicos, por tanto es posible que algunos nombres hayan variado en su lenguaje debido a la migración y mezcla de la población con otras culturas.

Los biotipos más frecuentes fueron árboles, seguido de hierbas y arbustos, resultado similar al obtenido por Levy et al. (2002) para la selva lacandona, aunque señalan en segundo lugar la frecuencia del uso de los bejucos, posiblemente debido a que el pueblo lacandón se dedica mayormente al manejo y conservación de la selva primaria, en comparación con las comunidades choles, quienes en los últimos años han incrementado el sistema de producción comercial de palma aceitera (Aguilar, 2014). En cambio, Toledo et al. (1995) destacan la importancia de las formas no arbóreas y la importancia relativa de los árboles, señalan que este fenómeno resulta diferente en los diversos hábitats; es decir, en las selvas primarias, los árboles útiles resultan más numerosos que las hierbas y arbustos; en tanto que, en las selvas secundarias la situación es justamente a la inversa. De igual manera, Neulinger et al. (2013) y Guillermo (2013) señalan para algunos agroecosistemas como huertos, milpa y potrero, mayor uso de especies herbáceas, con respecto a las leñosas, lo que fortalece también los resultados de Toledo et al. (1995).

No obstante, el pueblo ch'ol denota la importancia del uso de los árboles en el solar, lo cual es un indicativo de que el pueblo aún conserva el conocimiento del uso tradicional de biotipos representativos de selvas primarias, lo cual es muy importante para generar propuestas de conservación en el territorio. Las estructuras de las plantas más utilizadas fueron las hojas y los frutos, y aunque las demás partes son menos utilizadas, resaltan su importancia etnobotánica principalmente en los usos medicinal, ornamental y para la construcción; resultados similares señalan Toledo et al. (1995) y Levy et al. (2002).

De las 19 categorías antropocéntricas se reportó mayor número de especies usadas como comestible, medicinal, ornamental, construcción, combustible y sombra; datos similares fueron registrados por investigaciones en huertos familiares (VOGL et al., 2002; HURTADO y MORAES, 2010; MARIACA, 2012; HERNÁNDEZ-RUÍZ et al., 2014; SOL et al., 2016). Entre estas, Vogl et al. (2002), identificaron 11 categorías y presentaron usos representativos semejantes en huertos ch'oltzeltal-mestizo. De igual manera, Quique (2012) reconoce las categorías de comestible, ornamental y medicinal como las más importantes en huertos del Soconusco, Chiapas, e indica mayor uso ornamental sobre el comestible, lo cual fue reconocido también por Neulinger et al. (2013).

Basado en lo anterior, Toledo et al. (1995) indicaron que para los pueblos indígenas de la selva tropical, el uso de las plantas revela en este ecosistema una fuente muy importante de medicinas, alimentos, materiales de construcción, productos maderables y combustibles; señala que este patrón parece repetirse en casi todos los estudios etnobotánicos realizados en México, y parece constituir una regla observada en numerosos estudios realizados en grupos culturales de diferentes hábitats de Latinoamérica.

Por otra parte, la categoría comestible es la más representada, se registran formas de uso en comidas, bebidas, botanas, condimentos y postres; subcategorías que también fueron reconocidas por Toledo et al. (1995). En esta categoría, las familias más representadas son las Fabaceae, Solanaceae y Rutaceae, y la estructura más consumida son los frutos; semejante al estudio de Chávez et al. (2009), sobre las plantas no convencionales comestibles en Chiapas, quienes destacan el hecho de que las comunidades ch'ol conservan la tradición del uso de plantas comestibles silvestres y no convencionales, y el uso de las especies comunes establecidas dentro de la dieta mesoamericana (maíz, frijol, chile, calabaza); información que se mantiene para algunas comunidades indígenas de la región tropical (LEVY et al., 2002; CABALLERO et al., 2018).

\section{CONCLUSIONES}

El estudio etnobotánico en el Valle del Tulijá permitió revalorizar los saberes que posee el pueblo $c h$ 'ol sobre las especies vegetales presentes en el solar, además de comprender mediante el análisis de los aspectos socio-culturales, la importancia del conocimiento de los recursos naturales como elemento indispensable en sus sistemas de vida, lo que asegura la estabilidad del manejo tradicional y reafirma las formas de organización social e interpretación del territorio de este pueblo. Este estudio permitió identificar un importante número de especies de plantas, que presentan un valor de uso destacado dentro del sistema de vida de la población ch'ol.

El conocimiento tradicional ha sido un aporte indispensable para el aprovechamiento de las especies, y las zonas de manejo dentro del solar que influyen directamente en la conservación de la diversidad vegetal. El registro de nombre de especies en ch'ol genera el interés en la continuación de 
estudios sobre la clasificación taxonómica tradicional. Asimismo, esta investigación permite evidenciar y reafirmar la información generada en los diversos aportes que se han realizado sobre los huertos familiares, destaca la importancia que presentan para las poblaciones indígenas, así como el reconocimiento de que los conocimientos tradicionales aplicados en los procesos de producción agrícola, presentan una indudable estrategia de conservación y uso sustentable de los recursos naturales.

\section{AGRADECIMIENTOS}

Los autores agradecen al Consejo de Ciencia y Tecnología (CONACyT) por el financiamiento otorgado para la presente investigación, así como a las comunidades del Valle del Tulijá, Chiapas por su valiosa participación, y a los estudiantes de la Facultad Maya de Estudios Agropecuarios de la Universidad Autónoma de Chiapas Yesenia Guzmán López, Andrés Arcos Peñate, Jorge Alain López Sánchez y Cornelio Sánchez Vásquez actualmente profesionales, que apoyaron como traductores y asistentes en la realización de la misma.

\section{REFERENCIAS}

AGUILAR, J.C. La agricultura sostenible del Valle del Tulijá. Universidad Autónoma de Chiapas, Unidad de Divulgación Científica, Chiapas, México, 2014. 183 p.

AGUIRRE, J.; JARAMILLO, L. El papel de la descripción en la investigación cualitativa. Cinta de Moebio. Revista de Epistemología de Ciencias Sociales, Santiago, v. 53, p. 175-189, 2015.

AGUIRRE-RUÍZ, R.; TORRES-VÁZQUEZ, N.; VÁZQUEZ-TRUJILLO, E.; JIMÉNEZ-GUZMÁN, O.; RUIZ SÁNCHEZ, A.; VELÁZQUEZ-AVENDAÑO, J. Caracterización del subsistema de plantas medicinales en los patios traseros (paty otyoty) de la comunidad Amado Nervo, Municipio de Yajalón, Chiapas, México. Espacio Innovación más Desarrollo, México, v. 2, n. 2, p. 1-15, 2013.

ALEMÁN-SANTILLÁN, T. La otra diversidad chiapaneca. En: CONABIO - Comisión Nacional para el Conocimiento y Uso de la Biodiversidad Y Gobierno del Estado de Chiapas (org.). La biodiversidad en Chiapas: Estudio de Estado. México, 2013. p. 163-171.

ALTIERI, M.; NICHOLLS, C. Bases agroecológicas para una agricultura sustentable. En: ALTIERI, M.; NICHOLLS, C. (eds.). Agroecología: Teoría y práctica para una agricultura sustentable. Serie Textos Básicos para la Formación Ambiental, Programa de las Naciones Unidas para el Medio Ambiente, México, 2000. p. 13-40.

ANDRADE, G.; MEJÍA, G. La configuración del territorio $c h$ 'ol en torno a los caudales del río Tulijá. En: KAUFFER, M.; ESCOBAR, D. (coords.). De Chiapas a la Península de Yucatán. Universidad Autónoma de Chiapas, México, 2015. Capítulo n. 4, p. 99-117.

ARCOS LÓPEZ, N. Socialización lingüística intergeneracional: las narrativas en una familia $\boldsymbol{C h}$ 'ol de la ranchería Joljamil Grande, Tumbalá, Chiapas. 2013. 160 h. Tesis de Maestría (Maestría en Lingüística Indoamericana) - Centro de Investigaciones y Estudios Superiores en Antropología Social, México, D.F.

AULIE, H.W.; AULIE, E. Diccionario $c h$ 'ol de Tumbalá, Chiapas, con variaciones dialectales de Tila y Sabanilla. 3ed. Serie de vocabularios y diccionarios indígenas "Mariano Silva y Aceves", Instituto Lingüístico de Verano, México, 2009. v. 121, 258 p.

CASAS, A.; CAMOU, A.; OTERO-ARNAIZ, A.; RANGEL-LANDA, S.; CRUSE-SANDERS, J.; SOLÍS, L.; TORRES, I.; DELGADO, A.; MORENO-CALLES, A.; VALLEJO, M.; GUILLÉN, S.; BLANCAS, J.; PARRA, F.; FARFÁN-HEREDIA, B.; AGUIRRE-DUGUA, X.; ARELLANES, Y.; PÉREZ-NEGRÓN, E. Manejo tradicional de biodiversidad y ecosistemas en Mesoamérica: el Valle de Tehuacán. Investigación ambiental, Sección: Investigación, México D.F., v. 6 n. 2, p. 23-44, 2014.

CABALLERO-ROQUE, A.; ORANTES-GARCÍA， C.; MORENO-MORENO, R.; FARRERASARMIENTO, O. Huertos familiares en Chiapas. En: ORDÓÑEZ DÍAZ, M. (ed.). Atlas biocultural de huertos familiares en México: Chiapas, Hidalgo, Oaxaca, Veracruz y península de Yucatán. Universidad Nacional Autónoma de México, Centro Regional de Investigaciones Multidisciplinarias (CRIM), 2018. p. 121 165.

CDI - Comisión Nacional para el Desarrollo de los Pueblos Indígenas. Plan de Desarrollo de la Microrregión Valle de Tulijá, Chiapas, México. 2012. 126 p.

CONABIO - Comisión Nacional para el Uso de la Biodiversidad. Biodiversidad de especies en México. 2015. Disponible en: http://www.biodiversidad.gob.mx/especies/distribesp.html. Acceso en: 15 de ene. 2017.

CHÁVEZ, E.; ROLDÁN, J.; SOTELO, B.; BALLINAS, D.; LÓPEZ, E. Plantas comestibles no convencionales en Chiapas, México. Rev. Fac. Salud Pública Nutr., Nuevo León, v. 10, p. 1-12, 2009.

CONTRERAS-CORTÉS, L.; CASO-BARRERA, L.; ALIPHAT-FERNÁNDEZ, M.; MARIACA, R. Manejo de los agroecosistemas en la comunidad lacandona de Nahá, Chiapas. Etnobiología, México, v. 11, n. 3, p. 34 44, 2013. 
DE ÁVILA, A. La diversidad lingüística y el conocimiento etnobiológico. En: CONABIO - Comisión Nacional de la Biodiversidad (org.). Capital natural de México. Conocimiento actual de la biodiversidad. México, 2008, v. 1, p. 497-556.

FARRERA, S.; ORANTES, C. Plantas útiles multipropósitos en una comunidad del área de influencia de las reservas de la biosfera La Sepultura y El Ocote, Chiapas, México. Lacandonia, Tuxtla Gutiérrez, v. 9, n. 9, p. 37-44, 2015.

GUILLERMO GÓMEZ, M. Vegetación y etnobotánica de milpas y potreros en la región de los choles, en el municipio de Candelaria, Campeche. 2013. 161 h. Tesis de Maestría (Maestría en Ciencias Agropecuarias y Biotecnología) - Colegio de Postgraduados (COLPOS), Montecillo, México.

HERNÁNDEZ, M. Resumen, Usos de la biodiversidad. En: CONABIO - Comisión Nacional de la Biodiversidad y Gobierno del Estado de Chiapas (org.). La biodiversidad en Chiapas: Estudio de Estado. México, 2013. p. 162-164.

HERNÁNDEZ-XOLOCOTZI, E. Aspectos de la domesticación de plantas en México. En: RAMAMOORTHY, T.; BYE, R.; LOT, A.; FA, J. (comps.). Diversidad biológica de México. Universidad Nacional Autónoma de México, México, 1998. p. 715-735.

HERNÁNDEZ-RUÍZ, J.; JEREZ SALAS, M.; VÁZQUEZ DÁVILA, M.; VILLEGAS A. Uso antropocéntrico de especies vegetales en los solares de San Pedro Ixtlahuaca, Oaxaca México. Rev. Mex. Agroecosistemas, Oaxaca, v. 1, n. 1, p. 60-68, 2014.

HERNÁNDEZ, S. Cambios y continuidades en los solares mayas yucatecos. Un análisis intergeneracional de su configuración espacial en dos comunidades del sur de Yucatán. 2010. 169 h. Tesis de Maestría (Maestría en Ciencias en la Especialidad de Ecología Humana) - Instituto Politécnico Nacional, Mérida, México.

HURTADO, U.; MORAES, R. Comparación del uso de plantas por dos comunidades campesinas del bosque tucumano-boliviano de Vallegrande (Santa Cruz, Bolivia). Ecología en Bolivia, La Paz, v. 45, n. 1, p. 20-54, 2010.

INEGI - Instituto Nacional de Estadística y Geografía. Mapas, Marco Geoestadístico Nacional. 2017. Disponible en: https://www.inegi.org.mx/temas/mapas/mg/ Acceso en: 22 de nov. 2016.

JOHNS, T. Agrobiodiversidad, dieta y salud humana. En: JARVIS, D.; PADOCH, C.; COOPER, H. (eds.). Manejo de la agrobiodiversidad en los ecosistemas agrícolas. Columbia University Press, Biodiversity International, Italia, 2011. p. 404-430.

KAUFMAN, T.; JUSTESON, J. A preliminary Mayan Etymological Dictionary. Foundation for the Advancement of Mesoamerican Studies, Inc. (FAMSI). 2003. 1535 p. Disponible en: http://www. famsi. org/reports/01051/pmed. pdf. Acceso en: 16 de oct. 2017.

LERNER, T.; MARIACA, R.; IZABA, B.; GONZÁLEZ-JÁCOME, A.; KLEISSER, E. Aporte de alimentos del huerto familiar a la economía campesina ch'ol, Suclumpá, Chiapas, Etnobiología, México, v. 7, p. 30-44, 2009.

LEVY TACHER, A.; RIVERA, S.; MARTÍNEZ, M.; DURÁN, A. Caracterización del uso tradicional de la flora espontánea en la comunidad lacandona de Lacanhá, Chansayab, Chiapas, México. Interciencia, México D.F., v. 27, n. 10, p. 512-520, 2002.

LÓPEZ TOLEDO, J.; VALDEZ HERNÁNDEZ, J. Uso de especies arbóreas en una comunidad de la reserva de la biosfera La Sepultura, Estado de Chiapas. En: ENDARA AGRAMONT, Á.; MORA, A.; VALDEZ, J. (eds.). Bosques y Árboles del trópico mexicano. Universidad de Guadalajara, Prometeo Editores. México, 2011. p. 57-75.

LUNA-MORALES, C. Ciencia, Conocimiento tradicional y Etnobotánica. Etnobiología, México, v. 2, p. 120-135, 2002.

MARIACA MÉNDEZ, R. La complejidad del huerto familiar maya del sureste de México. En: MARIACA MÉNDEZ, R. (ed.). El huerto familiar del sureste de México. Secretaría de Recursos Naturales y Protección Ambiental del Estado de Tabasco, El Colegio de Frontera Sur, México, 2012. p. 7-97.

MARÍN-CORBA, C.; CÁRDENAS-LÓPEZ, D.; SUÁREZ-SUÁREZ, S. Utilidad del valor de uso en etnobotánica. Estudio en el Departamento de Putumayo (Colombia). Caldasia, Bogotá, v. 27, n. 1, p. 89-101, 2005 .

MELÉNDEZ, L.; TRABANINO, F.; CABALLERO, A. Tres Perspectivas en torno al uso comestible de las inflorescencias de las palmas pacay(a) y chapay(a) en Chiapas, México: enfoques paleoetnobotánico, nutricional y lingüístico. Estudios de Cultura Maya, México D.F., v. 41, p. 175-199, 2012.

MENDOZA, A. Caracterización del traspatio rural en localidades de la región de Los Llanos, Chiapas, México. 2015. 98 h. Tesis de Maestría (Maestría en Ciencias en Producción Agropecuaria Tropical) Universidad Autónoma de Chiapas, México.

MONTENEGRO, M.; LAGOS, T.; VÉLEZ, J. Agrodiversidad de los huertos caseros de la región andina del sur de Colombia, Rev. Cienc. Agric., Nariño, v. 34, n. 1, p. 50-63, 2017.

MONTEJO, B.; LÓPEZ, R.; GUZMÁN, J.; JIMÉNEZ, E.; MARTÍNEZ, E. Säkläji’b ty'añ ch'ol. Dirección de Educación Indígena, Gobierno Estado de Chiapas, México. 2001. 291 p. 
MORALES, M.; SALVATIERRA, B. Capital territorial del Valle de Tulijá: caso de los choles de Salto de Agua, Chiapas, México. Temas antropol. Revista Científica de Investigaciones Regionales, México, v. 34, n. 1, p. 11-35, 2012.

NEULINGER, K.; VOGL, C.; ALAYÓN-GAMBOA, J. Plant species and their uses in homegardens of migrant Maya and Mestizo smallholder farmers in Calakmul, Campeche, Mexico. J. Ethnobiol., Tacoma W.A., v. 33, n. 1, p. 105-124, 2013.

PHILLIPS, O. Some quantitative methods for analyzing ethnobotanical knowledge. En: ALEXIADES, M. (ed.). Selected guidelines for ethnobotanical research: a field manual. The New York Botanical Garden, U.S., 1996. p. 171-197.

PIGNATARO, A.; LEVY TACHER, S.; RIVERA, J.; TORAL, J.; ESPINOSA, M.; CARMONA, N. Silvopastoral systems of the Chol Mayan ethnic group in southern Mexico: Strategies with a traditional basis. J. Environ Manage., Kiel, v. 181, p. 363-373, 2016.

QUINTERO, I.; CUCHILlO, C.; CAMAYO, A.; MUYUY, E.; MUÑOZ, J.; ZARAGOZA, L.; RODRÍGUEZ, G.; ÁLVAREZ, L. El Tull o huerto ancestral de los indígenas Nasa de Cauca (Colombia). AICA, Córdoba, v. 6, p. 500-505, 2015.

QUIQUE, R. Cocoa, coffee and sugarcane farmers: an ethnobotanical characterization of their homegardens in the Soconusco region (Chiapas, Mexico). 2012. Tesis de Doctorado, University Montpellier II, France, El Colegio de la Frontera Sur, México.

RODRÍGUEZ-GALVÁN, G. Jardín tradicional. El traspatio de Los Altos de Chiapas. En: PEREZGROVAS, R.; RODRÍGUEZ, G.; ZARAGOZA, L. (eds.). El traspatio iberoamericano. Experiencias y reflexiones en Argentina, Bolivia, Brasil, España, México y Uruguay. Universidad Autónoma de Chiapas, México, 2011. p. 137-166.

RODRÍGUEZ-GALVÁN, G.; REISING, C.; MORONTA, M.; ÁLVAREZ, L.; ZARAGOZA, L. Estudio de sistemas ganaderos sustentables mediante un proceso metodológico estandarizado. AICA, Córdoba, v. 6, p. 255$265,2015$.

RZEDOWSKI, J. Vegetación de México. Comisión Nacional para el Conocimiento y Uso de la Biodiversidad (CONABIO), Edición digital, México, 2006. 505 p.

SANDOVAL, C. Investigación cualitativa. Programa de Especialización en teoría, métodos y técnicas de investigación social. Instituto Colombiano para el Fomento de la Educación Superior (ICFES), Editores LTDAARFO, Colombia, 2002.

SCHUMANN, O. La lengua chol, de Tila (Chiapas). Universidad Nacional Autónoma de México, Coordinación de Humanidades, México, v. 8. 1973.

SOL, Á.; GÓMEZ, E.; GARCÍA, E.; PÉREZ, A. Flora útil en el Ejido Sinaloa $1^{\text {a }}$ Sección, Cárdenas, Tabasco. Rev. Mex. De Cienc. Agric., México, v. 14, p. 2671-2681, 2016.

TOLEDO, V.; BATIS, A.; BECERRA, R.; MARTÍNEZ, E.; RAMOS, C. La selva útil: etnobotánica cuantitativa de los grupos indígenas del trópico húmedo de México. Interciencia, México, v. 20, n. 4, p. 177$187,1995$.

TORRES ROSAS, N. El solar: sitio de conservación de germoplasma y biodiversidad, en tres localidades del municipio de Cárdenas, Tabasco. 2010. 121 h. Tesis de Maestría (Maestría en Ciencias Producción Agroalimentaria en el Trópico) - Colegio de Posgraduados (COLPOS), Tabasco, México.

VENEGAS DURÁN, B. Arqueobotánica de Palenque, Chiapas. Una propuesta de investigación ante nuevos retos y nuevas perspectivas. Temas Antropol. Revista Científica de Investigaciones Regionales, Mérida, v. 39 , n. 1, p. $133-155,2017$.

VOGL, C.; VOGL-LUKASSER, B.; PURI, R. Tools and methods for data collection in ethnobotanical studies of homegardens. Field methods, U.S., v. 16, n. 3, p. 285-306, 2004.

VOGL, C.; VOGL-LUKASSER, B.; CABALLERO, J. Homegardens of Maya Migrants in the District of Palenque (Chiapas/Mexico). En: STEPP, J.; FELICE, S.; ZARGER, R. Ethnobiology and Biocultural Diversity. University of Georgia Press; Georgia, U.S., 2002. p. 631-647. 